\title{
Targeted Delivery System of Nanobiomaterials in Anticancer Therapy: From Cells to Clinics
}

\author{
Su-Eon Jin, ${ }^{1}$ Hyo-Eon Jin, ${ }^{2}$ and Soon-Sun Hong ${ }^{1}$ \\ ${ }^{1}$ Department of Drug Development, College of Medicine, Inha University, 3-ga, Sinheung dong, Jung-gu, \\ Incheon 400-712, Republic of Korea \\ ${ }^{2}$ Department of Bioengineering, University of California, Berkeley and Physical Biosciences Division, \\ Lawrence Berkeley National Laboratory, Berkeley, CA 94720, USA
}

Correspondence should be addressed to Soon-Sun Hong; hongs@inha.ac.kr

Received 25 November 2013; Accepted 25 December 2013; Published 19 February 2014

Academic Editor: Dong-Wook Han

Copyright (C) 2014 Su-Eon Jin et al. This is an open access article distributed under the Creative Commons Attribution License, which permits unrestricted use, distribution, and reproduction in any medium, provided the original work is properly cited.

Targeted delivery systems of nanobiomaterials are necessary to be developed for the diagnosis and treatment of cancer. Nanobiomaterials can be engineered to recognize cancer-specific receptors at the cellular levels and to deliver anticancer drugs into the diseased sites. In particular, nanobiomaterial-based nanocarriers, so-called nanoplatforms, are the design of the targeted delivery systems such as liposomes, polymeric nanoparticles/micelles, nanoconjugates, norganic materials, carbon-based nanobiomaterials, and bioinspired phage system, which are based on the nanosize of 1-100 $\mathrm{nm}$ in diameter. In this review, the design and the application of these nanoplatforms are discussed at the cellular levels as well as in the clinics. We believe that this review can offer recent advances in the targeted delivery systems of nanobiomaterials regarding in vitro and in vivo applications and the translation of nanobiomaterials to nanomedicine in anticancer therapy.

\section{Introduction}

Cancer is a worldwide disease with a leading cause of mortality, accounting for about 580,350 deaths, almost 1,600 people per day in 2013 from the statistical analysis of American Cancer Society in National Cancer Institute of the US [1]. About 1,660,290 of new cancer cases are also expected to be diagnosed in 2013. The 5-year relative survival rate is still somewhat low, at $68 \%$ for all cancers diagnosed between 2002 and 2008, although it has been up from $49 \%$ in the period from 1975 to 1977. For this reason, it is essential for targeted therapy for cancer to reduce adverse reactions and mortality rate and to save costs in clinical practice. Recently, targeted anticancer therapeutics such as monoclonal antibodies (mAbs) and tyrosine kinase inhibitors (TKIs) have been approved by the Food and Drug Administration (FDA) for the treatment of cancer [2]. The targeted therapy becomes an important element for the treatment of cancer as it helps to develop the anticancer therapeutics based on imaging and therapy (reducing the tumor size).
In this concept of targeted anticancer therapy, nanoplatforms are introduced with nanobiomaterial-based formulations or conjugation techniques in nanotechnology $[3,4]$. Nanotechnology is a nanoscale-based technique in the fields of biomedical applications of pharmacology, bioengineering, biology, and medicine [5]. It currently relies on definitions provided by the National Nanotechnology Initiative (NNI) as follows [6]: (1) development of research and technology at the atomic, molecular, or macromolecular levels, within the scale of nanosize of approximately 1 to 100 nanometer in range, (2) devices and systems that have novel properties and functions based on the nanobiomaterials, because of their small and/or intermediate size, and (3) ability to control or manipulate at the atomic level. A variety of nanoparticular systems span the range from a few nanometers to hundreds of nanometers. When such nanoparticle-based systems are usually applied in solving the clinical problems, we often use the term "nanoplatforms" [7].

Nanoplatforms have been developed to manufacture nanomedicines in preclinical and clinical studies for the 
administration of small molecules, genes, and peptides with improvement of given in vivo behavior [8-10]. Figure 1 shows the schematic diagrams of targeted delivery systems with nanoplatforms, such as liposome, polymeric micelle, nanoconjugate, gold nanoparticle, carbon nanotube, dendrimer, and phage-based nanoplatform. Several characteristics of an ideal tumor-targeted nanomedicine with nanoplatforms are presented for translational research of ideal anticancer therapeutics as follows: (1) increase of drug localization into the tumor by passive targeting or active targeting, (2) decrease of drug localization in sensitive, nontargeted, and normal tissues, (3) minimal drug leakage during transit to the target, (4) prevention of degradation and premature clearance of the drug, (5) retainment of the drug at the target site during the desired period, (6) facilitation of cellular uptake and intracellular trafficking, and (7) biocompatibility and biodegradability of nanoplatforms [10]. Table 1 illustrates the recently developed nanoplatforms for cancer targeting in preclinical studies. We introduce recent studies of targeted anticancer system in the part of "recently developed nanoplatforms for cancer targeting in preclinical studies."

For successful translational research of nanoplatforms in anticancer therapy, Doxil is an FDA approved drug for the treatment of ovarian cancer, which is a long-acting pegylated liposomal formulation of doxorubicin [11, 12]. This product is based on a study of liposomal drug delivery systems for over four decades overcoming the limitations of anticancer drug and liposomes such as poor stability and reproducibility $[13,14]$. It is one of the successful cases for the development of nanomedicines offering significant improvements over doxorubicin alone in clinical use. We deal with the translational research of nanomedicines in Table 2 and introduce the anticancer drugs approved by FDA in Table 3. Based on the ideal characteristics of nanoplatforms, they can be designed for the following: (1) sustained release of the drug, (2) passive accumulation of the tumor tissue, (3) ligand-based targeting of cell surface antigens or receptors with the modulation of endosomal uptake and membrane disruption, (4) drug release into the cytoplasm, and (5) protection from enzymatic degradation $[15,16]$. Additionally, in this review, we demonstrate the applications of targeted delivery systems from the cells to the clinics for anticancer therapy, diagnostics, nanoimaging, bimodal imaging, and real-time intraoperative imaging.

\section{Mechanism of Cancer Targeting by Nanoplatforms}

Many nanobiomaterial-based platforms have been designed and evaluated for drug targeting to cancers as shown in Figure 2. Most of these platforms are "passive targeting" concepts to improve the circulation time of the conjugated or encapsulated therapeutic drug such as liposomes, polymers, micelles, and nanoparticles. Solid tumors have blood vessels with enhanced vascular permeability and a lack of functional lymphatics, which allow extravasation of carrier materials with sizes of up to several hundreds of nanometers and are unable to eliminate extravasated nanomaterials. From the several reports, this EPR effect was shown even for the particles size of 1-2 $\mu \mathrm{m}$ (e.g., Lactobacillus sp., Salmonella sp., etc.), which could make the particles accumulated in tumor tissues [17, 18]. Therefore, long-circulating nanomedicines are able to be accumulated in tumors over time based on a mechanism known as the enhanced permeability and retention (EPR) effect $[19,20]$. The EPR effect is a unique phenomenon of solid tumors related to their anatomical and pathophysiological differences from normal tissues and is the most important strategy to improve the delivery of therapeutic agents to tumors for anticancer drug development. Examples of passively targeted nanoplatforms approved for clinical use are Doxil (Caelyx in Europe; pegylated liposomal doxorubicin), DaunoXome (nonpegylated liposomal daunorubicin), DepoCyt (nonpegylated liposomal cytarabine), Myocet (nonpegylated liposomal doxorubicin), Oncaspar (pegylated L-asparaginase), Abraxane (albuminbased paclitaxel), and Genexol-PM (paclitaxel-containing polymeric micelles, approved in Korea). In particular Doxil is a pegylated liposome-based, doxorubicin-loaded anticancer drug, which is FDA approved for the treatment of ovarian cancer as mentioned above. This drug has a severe cardiotoxicity although it overcomes poor stability and reproducibility of liposomes and improves a biodistribution of doxorubicin in tumor tissues by EPR effect. In addition, Abraxane is a Cremophor EL-free albuminbound paclitaxel. It reduced Cremophor EL-associated side effects of Taxol such as severe anaphylactoid hypersensitivity reactions, hyperlipidaemia, abnormal lipoprotein patterns, aggregation of erythrocytes, and peripheral neuropathy [21]. Next-generation nanomedicines for anticancer therapy are developing alternative approaches with recently developed nanoplatforms minimizing side effects of surfactants in formulations and targeting tumor tissues. Several additional passively tumor-targeted nanomedicines are currently in clinical trials (Table 2), and many other ones are in early and late-stage preclinical development [9, 22-25].

On the other hand, "active targeting" strategy indicates the use of targeting ligands like antibodies and peptides which are attached to drugs and drug delivery nanoplatforms for binding to receptor expressed at the target site. In this strategy, active targeting systems with ligands and antibodies need to be accumulated first in tumor tissues by EPR effect, and then active targeting could be achieved. Targeting ligands for actively targeting nanomedicines are improving cellular internalization via endocytosis-prone surface receptors, such as folate [26], galactosamine [27], EGF [28], and transferrin [29]. To date, targeting techniques for active targeting are advanced at the preclinical level, however, only antibody-based nanomedicines have been approved for clinical use (Tables 2 and 3). Zevalin (CD20-targeted ${ }^{90}$ Yttriumibritumomab tiuxetan), Bexxar (CD20-targeted iodine-131 tositumomab), Ontak (CD25-targeted diphtheria toxin-IL2 fusion protein), and Mylotarg (CD33-targetd gemtuzumab oxogamicin) have been successfully used for non-Hodgkin's lymphoma, T-cell lymphoma, and acute myeloid leukemia. In addition, it has necessitated the use of peptides to enable the 


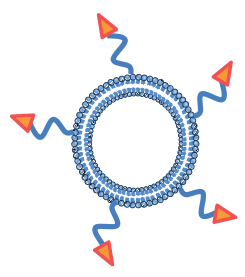

(a)

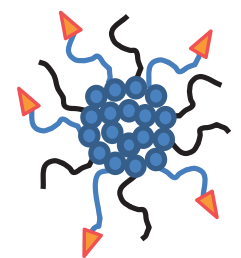

(b)

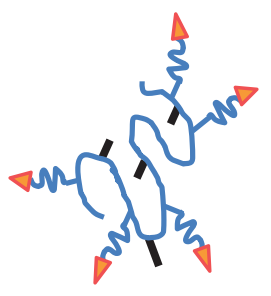

(c)

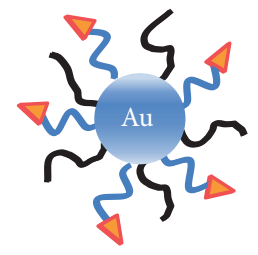

(d)

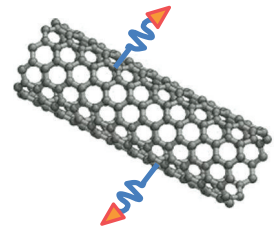

Targeting ligand

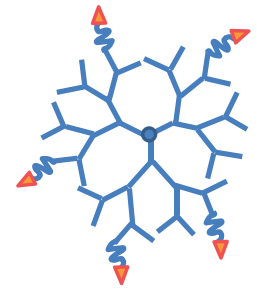

(f)

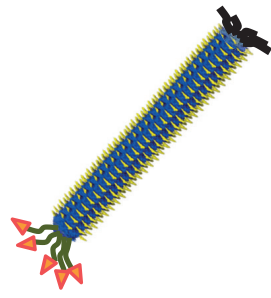

(g)

FIGURE 1: Schematic diagrams of the targeted delivery systems: (a) liposome, (b) polymeric micelle, (c) nanoconjugate, (d) gold nanoparticle, (e) carbon nanotube, (f) dendrimer, and (g) filamentous phage (M13, Fd).

cell internalization of cancer drugs such as cell-penetrating peptides, protein-transduction domains, oligoarginine, and TAT [30].

Active targeting to receptors, which are overexpressed by angiogenic endothelial cells, can reduce blood supply to tumors that deprive the tumor cells from oxygen and nutrients in solid tumors. Ligands used for drugs and drug delivery platforms to tumor vasculature include antibody fragment L19 [31], as well as several cyclic and linear derivatives of the oligopeptide RGD and NGR, which bind to angiogenic endothelium through the integrins $\left(\alpha_{2 \mathrm{~b}} \beta_{3}, \alpha_{\mathrm{v}} \beta_{3}\right.$, and $\left.\alpha_{5} \beta_{1}\right)$ and aminopeptidase-N (CD13), respectively [32, 33].

Although active targeting systems are extensively studied to develop anticancer therapeutics, there has been a less example of clinical use compared to passive targeting systems. Discussing these problems to overcome, active targeted nanoplatforms are physicochemically unstable for blood circulation in the body and hard to be accumulated in tumor tissues due to their size of conjugated targeting ligands of formulations (e.g., monoclonal antibodies) [34]. In addition, active targeting systems can be eliminated by nanoparticular surface opsonization (nonspecific protein adsorption) and nanoparticular uptake and retention to reticuloendothelial systems resulting in poor efficiency of active targeting system in vivo [35]. Based on these fundamental problems and technical barriers, multifunctionality, targetability, and stability are necessary to overcome the low efficiency of active targeting nanoplatforms.

\section{Nanoplatforms of Anticancer Therapeutics and Application in Clinical Trials}

Targeted delivery systems of nanobiomaterials are currently in the process of developing nanoscale-sized platforms or surface modification of nanoplatforms with active targeting ligands that support multifunctionality due to poor solubility of anticancer drugs or toxic effect of drugs on noncancerous cells and tissues [36, 37]. Most of the anticancer drugs are poorly soluble or insoluble in water. Thus, organic solvents or toxic surfactants are usually applied for the formulation of anticancer therapeutics even used in clinics. In addition, anticancer drug alone has a toxic effect on normal cells and tissues without target specificity $[38,39]$. Therefore, several nanoplatforms in $100 \mathrm{~nm}$ diameter based on nanobiomaterials have been used to administer the anticancer drugs to minimize the adverse toxicity and to maximize the drug effect within the therapeutic index [40].

Nanoplatforms offer solubility of poorly soluble anticancer drugs in water preparing soluble suspensions with the minimum need for organic solvents and surfactants which cause toxicity $[40,41]$. These platforms are accumulated in the tumor tissues by the EPR effect, as mentioned above. The nanoplatforms are also used in the targeted delivery systems of active targeting using the decoration with receptor-targeted ligands or tumor antigen sensitive antibodies together with anticancer drugs [22, 37, 42-44]. The anticancer drugs encapsulated into the nanoplatforms, specifically liposomal nanoplatforms and polymer-based nanoplatforms, are undergoing clinical trials $[43,45,46]$. Table 2 shows the nanomedicines of anticancer drugs in clinical trials.

3.1. Doxorubicin. Doxorubicin, a very effective anticancer drug, is widely used in the treatment of breast, ovarian, bladder, and lung cancers [47]. Mechanism of action of doxorubicin is the blocker of topoisomerase II, which is an important enzyme in the DNA replication process that unwinds the DNA helix. The mechanisms of doxorubicin 


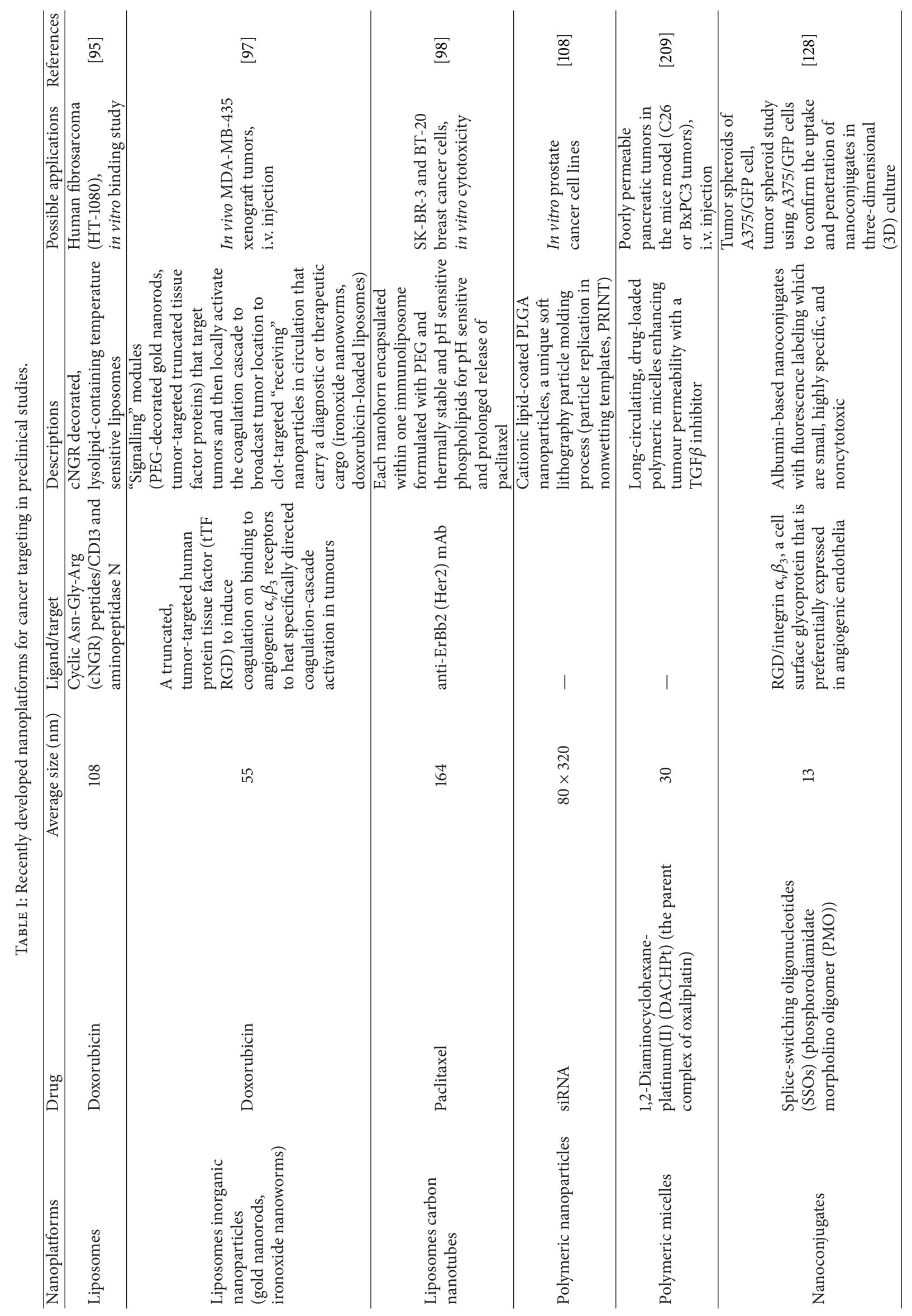




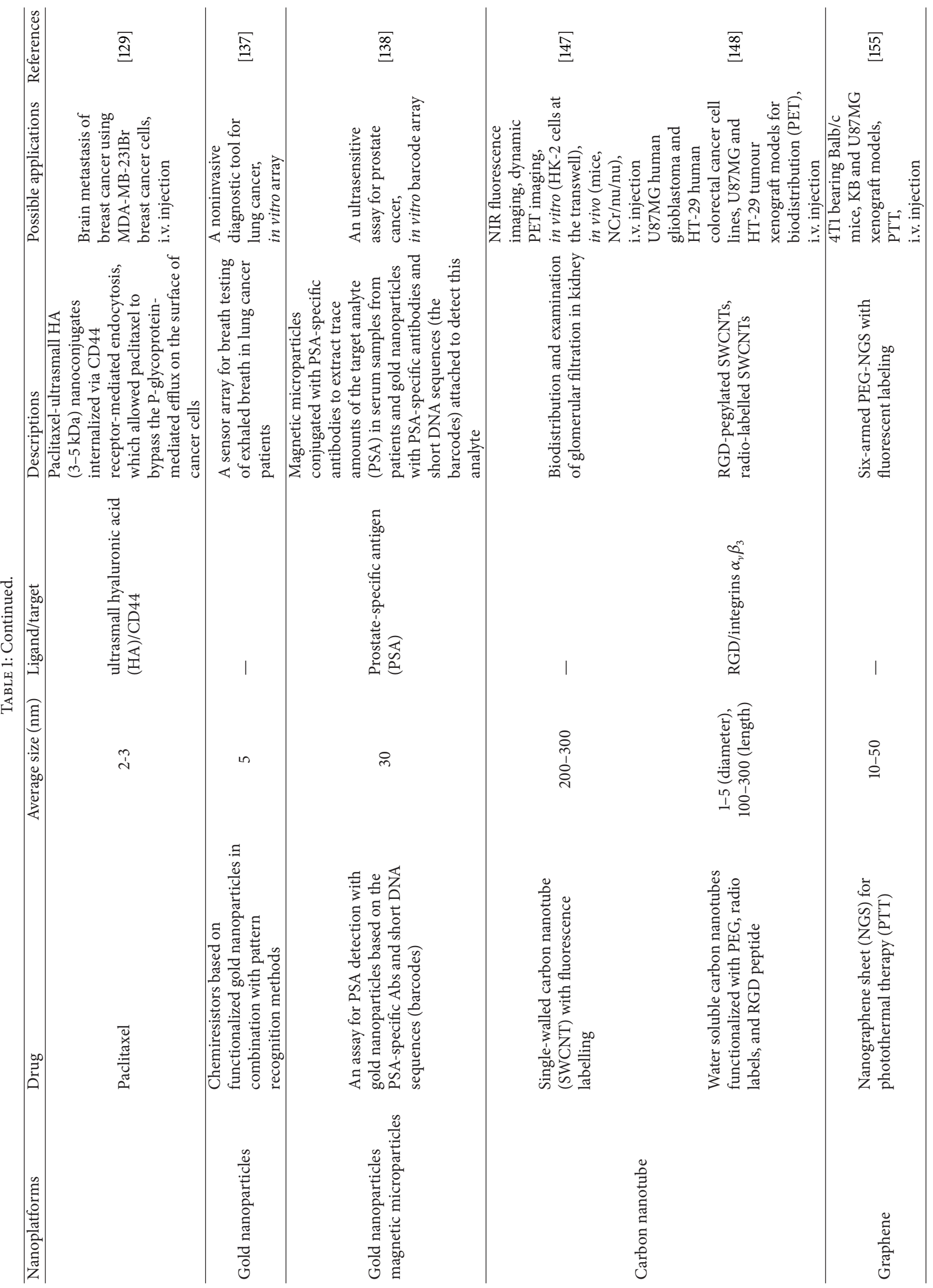




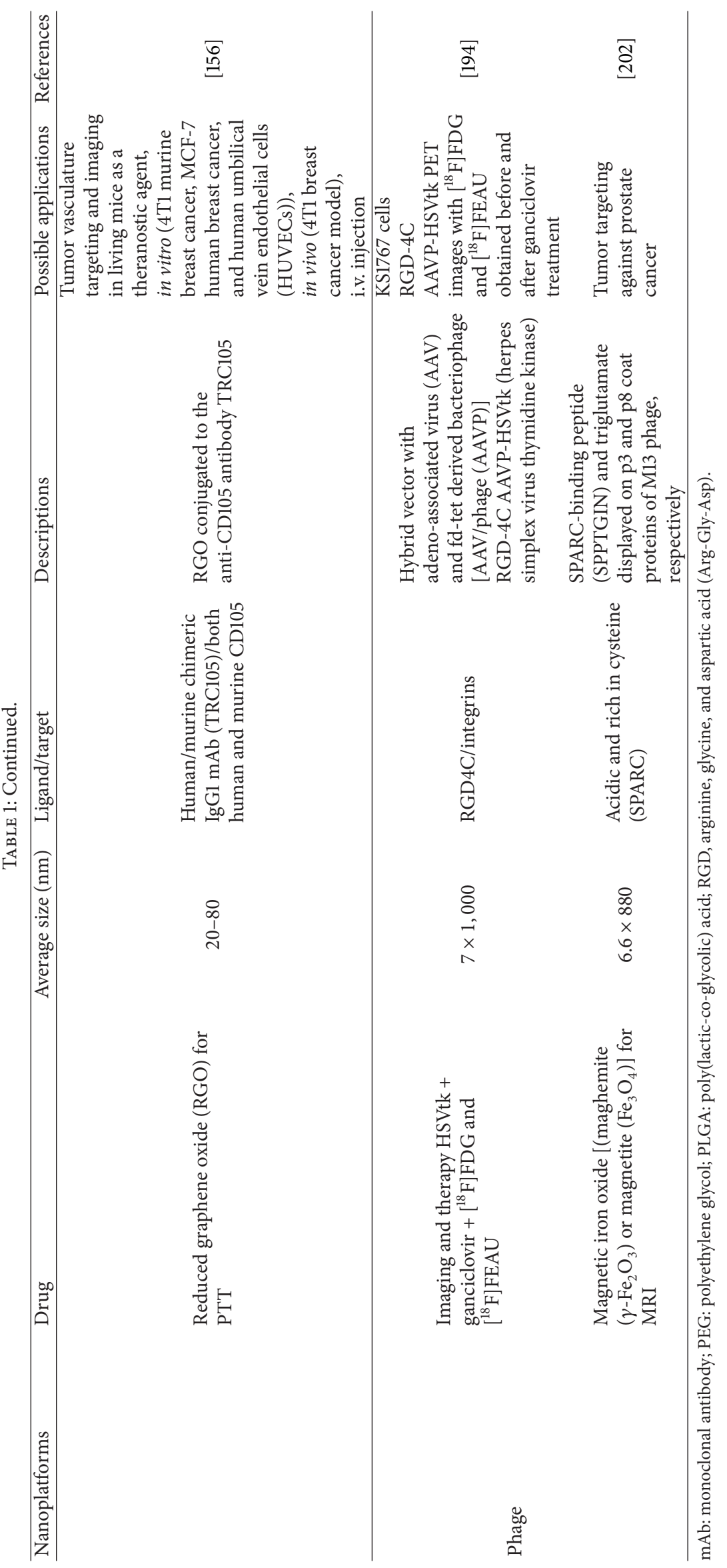




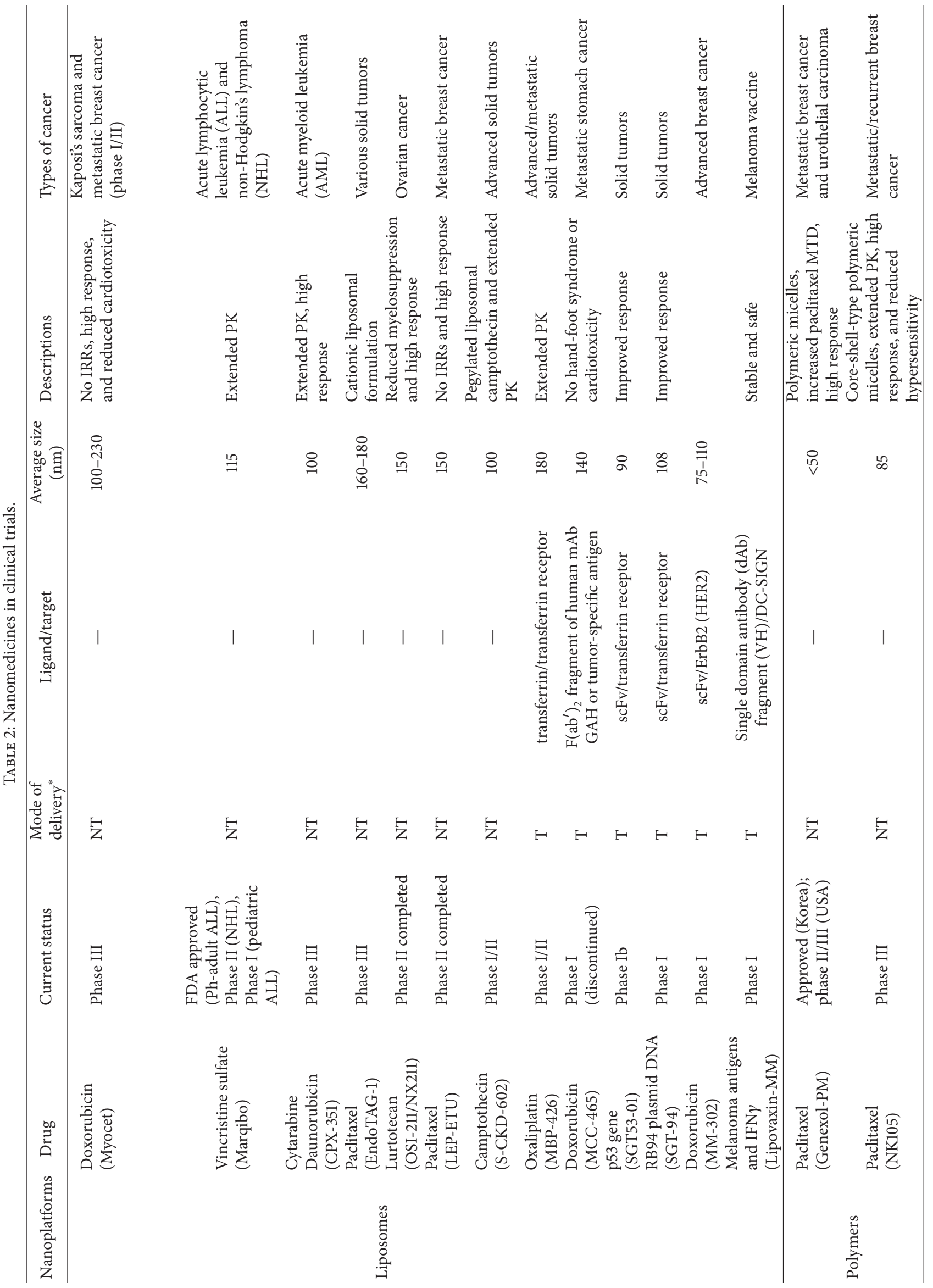




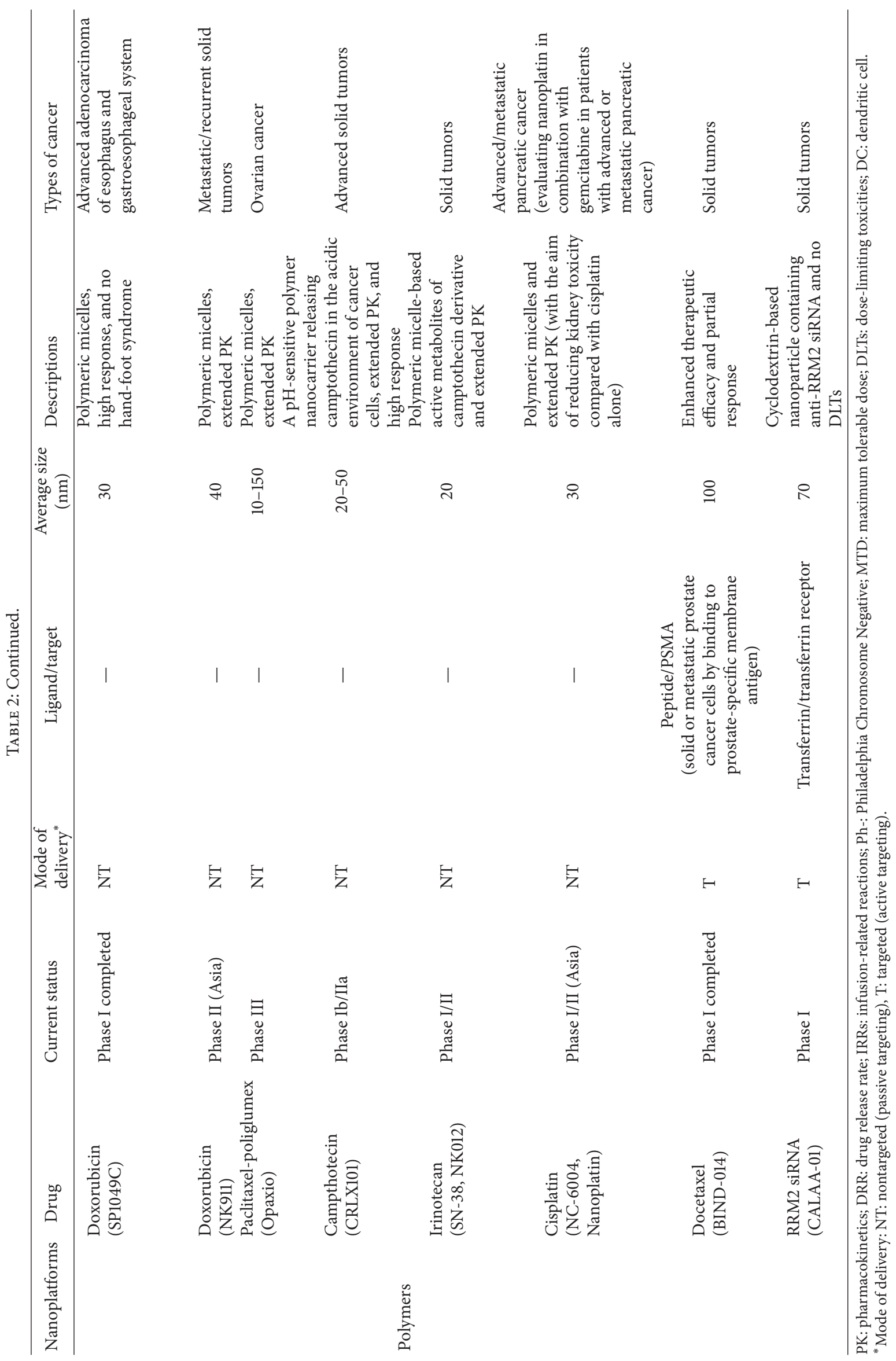




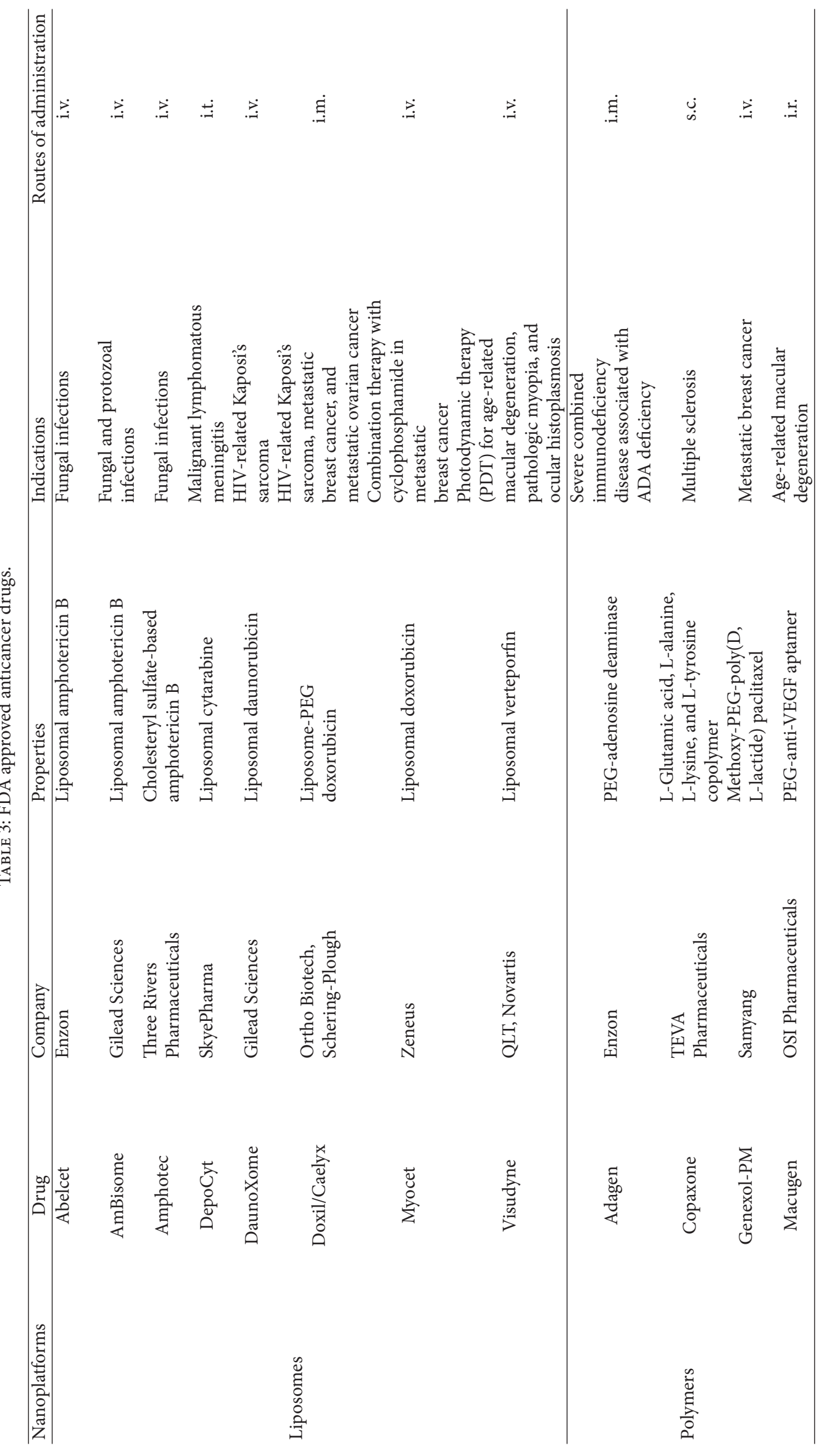




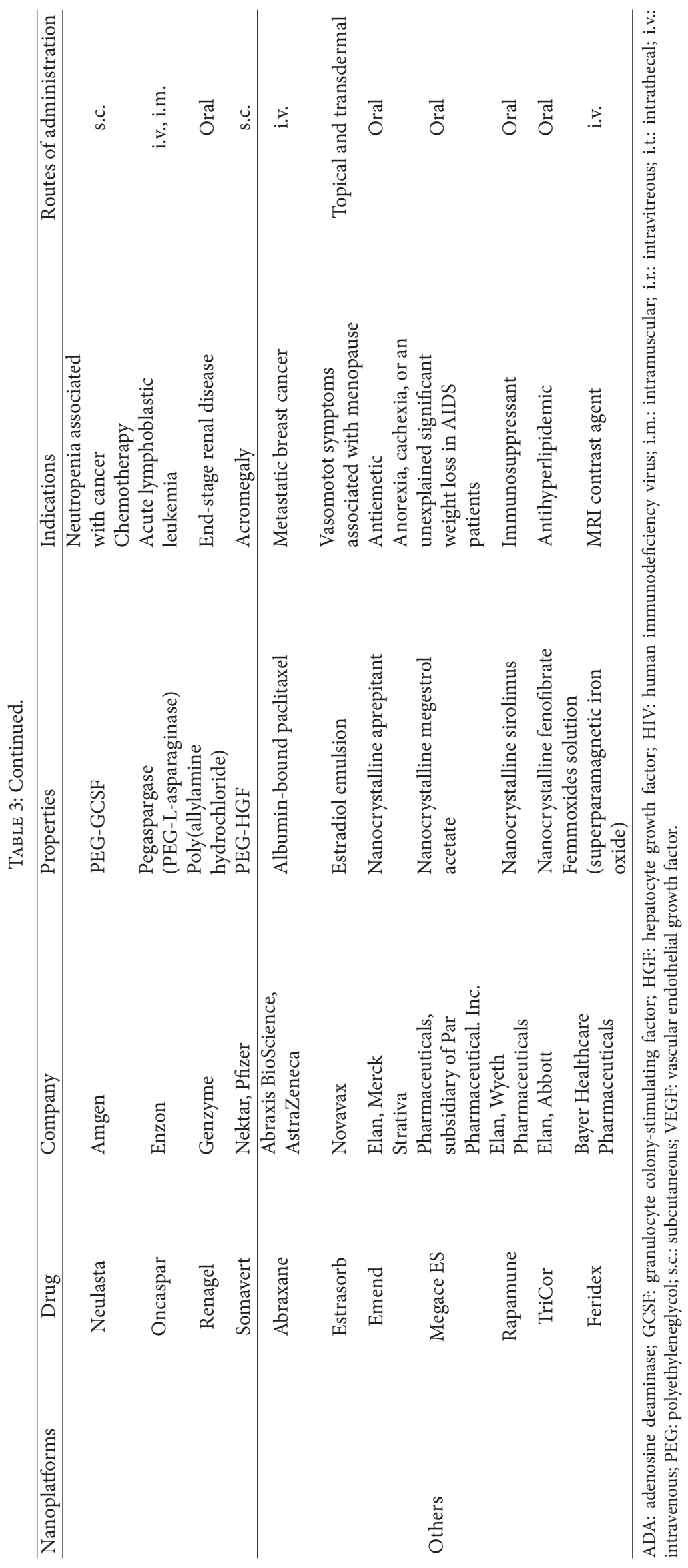




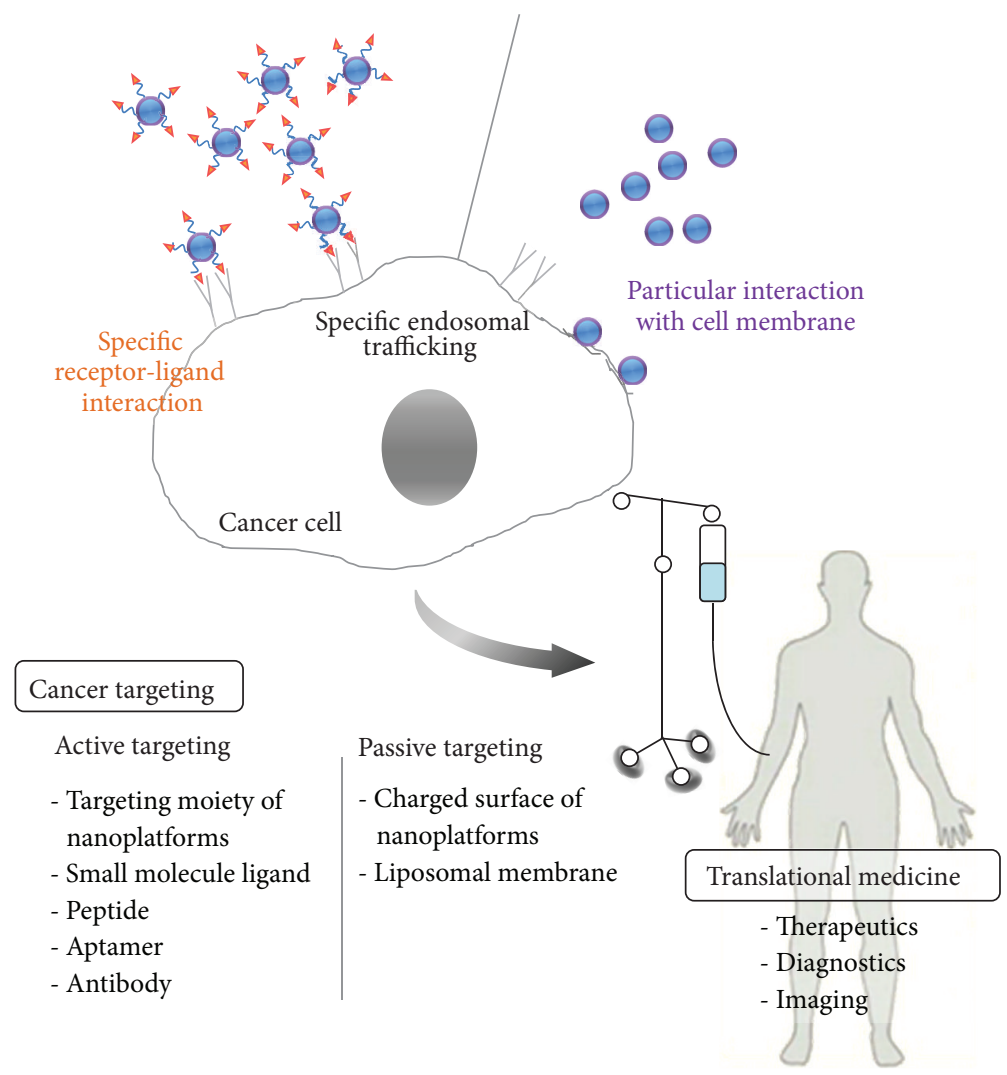

FIGURE 2: Overview of cancer targeting strategies from the cells to the clinics. This overview illustrates the most clinically relevant targeting strategies for anticancer therapy: passive targeting and active targeting. Passive targeting strategy is defined as the accumulation of nanoplatforms at the cancer cells by EPR effect, which uses nanoplatforms without targeting moieties. In this way, particular interaction will be proceeded to be internalized onto the cancer cells. On the other hand, active targeting strategy means the ligand-targeted or the receptor-mediated approach based on the cancer-specific targeting moieties of nanoplatforms, which interact with the specific receptorligand interaction on the cancer cells. Based on the cancer targeting strategies, translational medicine will be developed for the diagnostics, therapeutics, and imaging.

also include DNA cross-link and ROS generation besides inhibiting topoisomerase II. Based on these mechanisms of action, doxorubicin has a potent antitumor activity in tumor cells inducing cell death. However, doxorubicin is associated with the severe side effects on the heart including irreversible myocardiotoxicity and fatal congestive heart failure [48]. To decrease the toxicity of doxorubicin, nanoplatforms were introduced to enhance pharmacokinetic parameters with an accumulation of drugs in the tumor tissues, thereby minimizing the cardiotoxicity. Liposomes and polymeric nanoplatforms were studied and developed as nanomedicines via an intravenous route. Myocet, MCC-465, MM-302, SP1049C, and NK911 are the liposomal or polymeric nanopaltforms of doxorubicin in the clinical trials [49]. Myocet, MCC-465, and MM-302 are based on the liposomal nanoplatforms at the range of $100-140 \mathrm{~nm}$ in diameter [50]. In the cases of MCC-465 and MM-302, the target-specific ligands of $F\left(a b^{\prime}\right)_{2}$ fragment in human $\mathrm{mAb}$ GAH or tumor-specific antigen and scFv/ErbB2 (HER2) are incorporated into the liposomal nanoplatforms, respectively. In other words, SP1049C and NK911 are used in the polymeric micelles. SP1049C is a Pluronic-based micellar formulation of doxorubicin [51] and
NK911 is a core-shell-type polymeric micellar nanoplatform of doxorubicin which consists of a block copolymer of PEG (m.w., 5000) and poly(aspartic acid) (30 units) conjugated with doxorubicin [52]. Doxorubicin is entrapped into the highly hydrophobic inner core of the polymeric micellar nanoplatforms.

3.2. Paclitaxel. Paclitaxel is a chemotherapeutic agent for the ovarian, breast, and lung cancers as well as Kaposi's sarcoma [53]. It is a mitotic inhibitor with a stabilizing activity of the microtubule assembly interfering the normal breakdown of microtubules during cell division. Paclitaxel was originally extracted from the Pacific yew tree, Taxus brevifolia. Bristol-Myers Squibb commercially developed paclitaxel, a famous trademark, Taxol. However, paclitaxel itself has severe adverse responses such as peripheral sensory neuropathy $[54,55]$, anaphylaxis, and hypersensitivity reactions due to its solubilizing materials (Cremophor EL and ethanol) [55]. Therefore, the development of nanoplatforms is essential to overcome these problems of formulation for the improvement of pharmacokinetic parameters and the toxic adverse 
reactions in the normal tissues [56]. EndoTAG-1 [57], LEPETU [58], Genexol-PM [59], NK105 [60], and Opaxio [61] are developed as paclitaxel-loaded nanoplatforms undergoing clinical trials. EndoTAG-1 and LEP-ETU are based on the liposomal nanoplatforms. In particular, EndoTAG-1 is based on the cationic liposomal formulation, which can interact with the newly developed and negatively charged endothelial cells in the disease states for the growth of tumor blood vessels targeting the blood supply to the tumor cells [57]. Polymeric nanoplatform-based paclitaxel formulations are also developed such as Genexol-PM, NK105, and Opaxio. Paclitaxel is conjugated to poly(l-glutamic acid) (PGA) in Opaxio [61], monomethoxy poly(ethylene glycol)-blockpoly(D,L-lactide) (mPEG-PDLL) in Genexol-PM [59], and NK105 polymers of PEG as the hydrophilic segment and modified polyaspartate as the hydrophobic segment in NK105 [62], respectively.

3.3. Platinum-Based Anticancer Drugs. Cisplatin (cisplatinum or cis-diamminedichloroplatinum (II)) is also used in chemotherapy, which is a platinum-based anticancer drug for the treatment of various cancers including sarcomas, some carcinomas (e.g., small cell lung cancer and ovarian cancer), lymphomas, and germ cell tumors $[63,64]$. Mechanism of action in platinum-based anticancer drug is DNA crosslinking to interfere with the cell division by mitosis triggering apoptosis or cell death. Oxaliplatin and carboplatin are also included in platinum-based anticancer therapeutics. In clinical trials, oxaliplatin (MBP-426) [65] and cisplatin (NC-6004, Nanoplatin) [66] are studied for their applicability to advanced/metastatic solid tumors. In particular, Nanoplatin in combination with gemcitabine is evaluated for the treatment of advanced/metastatic pancreatic cancers. MBP-426 is reported as an oxaliplatin-based liposomal nanoformulation with the surface modification of transferrin targeting transferrin receptors in disease states. In the case of polymeric nanoplatforms, Nanoplatin, a polymeric micellebased cisplatin of $30 \mathrm{~nm}$ in diameter, is studied for its kidney toxicity reduction capabilities compared to cisplatin alone.

3.4. Camptothecins. Camptothecin and irinotecan, a watersoluble derivative of camptothecin, are cytotoxic alkaloids isolated from Camptotheca acuminate [67]. The target of these camptothecins and their derivatives is topoisomerase I to inhibit the replication in the cells. They bind to the topoisomerase I and DNA complex generating a stabilized ternary complex to prevent DNA religation and to cause DNA damage resulting in apoptosis. Camptothecin and its derivative are limitedly used due to lipophilicity and instability of the lactone ring structure by hydrolysis despite their superior anticancer activity [68]. Therefore, the nanoplatform-based camptothecin (S-CKD-602 [69] and CRLX101 [70]) and irinotecan (SN-38, NK012 [71]) are developed undergoing clinical trials. S-CKD-602 and CRLX101 are used in the nanoplatforms of a pegylated liposome and a polymeric micelle, respectively. In the case of irinotecan (SN38 , NK012), it is a polymeric micelle-based active metabolite of camptothecin to exploit the EPR effect in the diameter size of $20 \mathrm{~nm}$. This system is constructed in an amphiphilic block copolymer, PEG-PGlu (SN-38), by the self-assembly in the aqueous media. NK012 had an antitumor activity with tolerance that included partial responses and several occurrences of prolonged stable disease across a variety of advanced refractory cancers in the clinical studies.

3.5. Gene Therapy. Plasmid DNA and siRNA for cancer gene therapy are also used for the treatment of cancers in the clinical trials. The aim of gene therapy is to kill the cancer cells blocking the transduction of the tumor cells or inhibiting the disease-induced proteins without any damage to the normal cells, using cancer-specific genetic materials [72]. For the current clinical trials, p53 gene (SGT53-01) [73], RB94 plasmid DNA (SGT-94) [74], and RRM2 siRNA (CALAA01) [75] are studied for the treatment of solid tumors. These materials are based on tumor-specific nanoplatforms, which are the liposomal (SGT53-01 and SGT-94) or cyclodextrinbased nanoparticular (CALAA-01) systems decorated with a target ligand of transferrin receptors in the tumor cells. These nanoplatforms are necessary in delivering the genetic materials into the tumor cells due to the instability in the biological fluids (e.g., enzymatic digestion) and the hydrophilicity of genetic materials (e.g., poor uptake to cells).

\section{Recently Developed Nanoplatforms for Cancer Targeting in Preclinical Studies}

4.1. Liposomes. Liposomes have been developed as bioinspired nanoplatforms, which define the phospholipid bilayer showing the properties of the cell membrane [76, 77]. The liposomal nanoplatform can carry the hydrophilic drug at the aqueous core inside the bilayer and the hydrophobic drug at the lipid membrane. This nanoplatform has a lot of advantages for the delivery system with a great attention given their biocompatibility and targetability from the in vitro characterization to translational research [50, 78, 79]. For cancer targeting, liposomes are extensively used as passive targeting agents and ligand-mediated or stimuli-sensitive targeting agents (active targeting agents) [80]. Based on their biocompatibility, liposomes have been early developed as nanoplatforms to transfer small molecules in the tumor tissues [14]. Various anticancer drugs such as doxorubicin, doxorubicin derivatives, paclitaxel, and platinumbased anticancer drugs are usually loaded into the liposomal platforms (Table 1). These are 100-200 nm in average size, which makes liposomes take a trip into the tumor tissues.

Liposomes can also be used in the targeted delivery system with several modification of the liposomal surface design [81-84]. Cancer targeting systems are rapidly used in broad applications for the tumor-specific ligands $[85,86]$ or tumorassociated antigens [87] although liposomes are possible to be used in anticancer therapy as the delivery carriers for passive cancer targeting. The cancer-targeted therapy with targeting ligands includes leukocyte differentiation antigen (CD33) for acute myeloid leukemia [88], GD2 for neuroblastoma [89], and the folate receptor for wide human tumors [90, 91]. In addition, integrins [92], vascular endothelial growth 
factor receptors (VEGFR) [93], and CD13/aminopeptidase $\mathrm{N}$ [94] are also used as the targeting ligands. Liposomes with cyclic Asn-Gly-Arg (cNGR) peptide targeted to CD13 were currently formulated with lysogenic lipids-loaded doxorubicin for the potential treatment of human fibrosarcoma [95]. These systems are not only ligand-targeted systems for cancer metastasis, but also the hyperthermia-targeted systems for tumor hyperthermia, which regulates the release of doxorubicin specifically in the hyperthermal tissues for cancer targeting.

Liposomes can also be used as one of the components in the nanohybrid systems [96]. A study by Von Maltzahn et al. reported a complexed nanohybrid system for the treatment of human breast cancer in the xenograft tumor model of MDA-MB-435 cells [97]. This system is the complex of "signaling modules" to activate and broadcast the tumor location and "receiving modules" to carry the nanoplatformbased diagnostic agents and therapeutic drugs. They used the doxorubicin-loaded liposomes as therapeutic cargos with iron oxide nanoworms as the diagnostic agents in circulation. In addition, for the activation of doxorubicin-loaded liposomal cargos, PEG-decorated gold nanorods and tumortargeted truncated tissue factor proteins (tTF-RGD) were previously introduced as the signaling modules to induce the heat specifically directed coagulation on binding to the angiogenic receptors in tumors before adding the doxorubicinloaded liposomes. This system can be applied for the targeted theranostic agents to detect and treat the tumors together. Liposomes can also be used together with carbon nanotubes [98, 99]. Huang et al. reported carbon nanotubes encapsulated into the liposomes, which carried paclitaxel and antiErBb2 (Her2) mAb for this system SK-BR-3 and BT-20 breast cancer cells and checked the in vitro cytotoxicity [98]. This system can also be applied to theranostics for the potentiality of diagnostics and therapeutics.

4.2. Polymeric Nanoparticles/Micelles. Polymers are innovative nanobiomaterials to be engineered for the delivery system of drugs, genes, and peptides [41, 100]. Polymeric nanoparticles or micelles are easily prepared to be nanosized with various designs, which are relatively smaller in size compared to lipid-based formulations that generally range in size of 1-50 nm. Polymers can be used to carry multiple ligands for cancer targeting and imaging molecules for cancer diagnostics with a simple conjugation-based structural modification [101]. Among the polymers, PEG and poly(lactic-coglycolic) acid (PLGA) are extensively used as biocompatible polymers for translational medicine $[102,103]$.

PLGA is one of the most widely used polymers due to its biocompatibility, which is degraded to the monomers of lactic acid and glycolic acid in the body [104]. PLGA was approved by US FDA and European Medicine Agency (EMA). PLGAbased nanoparticles are successfully applied for drug delivery of the biomedical approaches for the development of translational medicine [105]. For example, paclitaxel-encapsulated PLGA nanoparticles were formulated with a strong enhancement of the cytotoxic effect of paclitaxel on the tumor cells in vitro and in vivo compared to commercial formulation,
Taxol [106, 107]. Hasan et al. published the cationic lipidcoated PLGA nanoparticles with a unique soft lithography particle molding process, which was applied for the delivery of siRNA in the prostate cancer cells [108]. In this study, they coated the PLGA nanoparticles with cationic lipid for a successful internalization to the cells based on a chargecharge interaction of the cell membrane and nanoparticles because PLGA has a negative charge. Based on the addition of targeting ligands like cLABL (ICAM-1 targeting) [109], folate (folate receptor) $[110,111]$, prostate-specific receptor antigen (prostate-specific receptor targeting) [112, 113], RGD (integrins $\alpha_{v} \beta_{3}$ ) $[114,115]$, and AS1411 (nucleolin targeting) $[116,117]$, PLGA nanoparticles have the potential to improve drug efficacy via target specificity of nanoplatforms in vitro and in vivo.

Although these nanoparticles have an effective function in cancer therapy in preclinical trials, these nanoparticles can be removed in circulation based on the biological barrier and reticuloendothelial system (RES), which performs an opsonization to macrophages that are internalized by phagocytosis [118]. PEG is the most commonly used polymeric moiety for the surface modification of nanoplatforms [119]. Nanoparticles can be usually decorated with targeting ligands conjugated with PEG. PEG originally has a function to make nanoparticles sterically stable for the prolonged circulation in the blood after administration. This method is called "pegylation" [120]. It demonstrates the hydrophilic moiety of PEG on the particular surface in the nanoplatforms, which provides the steric hindrance of a particular system to be shielded against the RES system for a prolonged delivery of the drug. Aravind et al. studied the long-circulating, drug-loaded polymeric micelles enhancing tumor permeability with a TGF $\beta$ inhibitor in the poorly permeable pancreatic tumors in a murine model of the C26 or BxPC3 tumors [116]. They loaded 1,2-diaminocyclohexaneplatinum(II)(DACHPt), which is the parent complex of oxaliplatin, in the polymeric micelles of PEG-b-poly(glutamic acid) (PEG-b-P(Glu)) copolymer and $\mathrm{P}(\mathrm{Glu})$ homopolymer. After the size-based screening of permeability into the tumors, $30 \mathrm{~nm}$ sized micelles had a relatively enhanced tumor permeability, when transforming growth factor $\beta$ was administrated together with the micelles.

4.3. Nanoconjugates. Nanoconjugates have extensively been studied as the smart nanoplatforms with active functional groups to prepare the covalent binding for the anticancer therapeutics [121]. Polymeric nanoconjugates are generally synthesized with a simple conjugation of the functional groups in the polymer such as $-\mathrm{OH},-\mathrm{COOH}$, and $-\mathrm{NH}_{2}$. These steps are stepwise reactions, which need to avoid uncontrollable side chemical synthesis. Nanoconjugates include the nanoplatforms of polymeric nanoparticles/micelles and other nanoparticles based on the characteristics of conjugation technique to prepare the nanoconjugate platforms using the functional groups of nanobiomaterials. Polymer-drug conjugates $[122,123]$ or mAb conjugates [42] were reported for targeted anticancer therapy. Xiong et al. introduced the cisplatin-based poly $(\gamma, 1$-glutamic acid)-citric acid-based nanoconjugates [123], and Segal 
and Satchi-Fainaro illustrated the polymeric nanoconjugatebased therapeutics with favorable polymers such as $\mathrm{N}$-(2hydroxypropyl)methacrylamide (HPMA), polyglutamic acid (PGA), and $\beta$-poly(L-malic acid) (Polycefin) [122]. In addition, Julien et al. showed $\mathrm{mAb}$-targeted nanobiomaterials for anticancer therapy [42]. They described the mAb-drug conjugates with an introduction of $\mathrm{mAb}$-based therapeutics such as CD20 (Rituxan for B-cell lymphoma), HER2 (Herceptin for breast cancer), VEGF (Avastin for colon, lung, breast, and renal cancer), and EGFR (Erbitux for colon and lung cancer).

These platforms have more advantages than other nanoplatforms, like micelles and liposomes, in the way that they are small in size and chemically stable in biological fluid. For this reason, these platforms are easily applied to the functionalization of multiple-target ligands or theranostic agents with polymers, peptides, proteins, or other nanoparticles. For example, folate is a widely used ligand for cancer targeting, which can have an interaction with the overexpressed folate receptors in cancer states [124]. Folate receptors are highly overexpressed in epithelial, ovarian, cervical, breast, lung, kidney, colorectal, and brain tumors while they are restrictedly expressed in the normal tissues, such as the lung, kidney, placenta, and choroid plexus, which are limited to the apical surface of the polarized epithelia $[124,125]$. Folate has a lot of advantages to be used for a targeting ligand through the conjugation to nanobiomaterials based on its small molecular weight $(441 \mathrm{Da})$ and the easy preparation method of folate-linked nanobiomaterials due to the stability of folate over a broad range of temperatures and $\mathrm{pH}$ values [126]. Zwicke et al. focused on the folate-based nanoconjugates for anticancer therapy with doxorubicin or paclitaxel and cancer imaging with other contrast agents like gold nanoparticles, iron oxide nanoparticles, or carbon nanotubes [127].

The nanoconjugate systems can be the effective targeting nanoplatforms of macromolecules to the tumor tissues based on their ultrasmall size although they carried the ligandbased targeting systems of cell surface antigens, peptides, or polymers. The albumin-based nanoconjugates were introduced by Ming et al. [128], which carried phosphorodiamidate morpholino oligomer (PMO) type splice-switching oligonucleotides (SSOs) with a RGD peptide for integrin $\alpha_{v} \beta_{3}$, a cell surface glycoprotein, as an active targeting ligand and a fluorescence label. This system was very small based on $13 \mathrm{~nm}$ of size. It had a high specificity without cytotoxicity. In particular, it was applied to the tumor spheroids of A375 cells to check the uptake and penetration of the albumin-based nanoconjugates into the three-dimensional (3D) cultures. In addition, these nanoconjugates can overcome the drawbacks of conventional chemotherapy such as drug toxicity to normal cells and cancer drug resistance by specific targeting and activating the cancer cells via the multiple decorations of target ligands on the nanoconjugates. Mittapalli et al. also reported the paclitaxel-ultrasmall hyaluronic acid (HA) nanoconjugates [129], which is a CD44 receptor targeting system for the treatment of brain metastasis of breast cancer in a model of MDA-MB-231 breast cancer cells. They used an ultrasmall $\mathrm{HA}(3-5 \mathrm{kDa})$ as a target ligand to interact with CD44 receptors on the surface of the cancer cells. In addition, this ultrasmall HA-mediated cellular uptake of paclitaxel avoided the P-glycoprotein-mediated efflux showing the drug resistance in cancer cells. In particular, this system exhibited a small size of 2-3 nm, like a single molecule of the nanoconjugates, and can self-assemble into larger particles.

4.4. Inorganic Nanoparticles-Iron Oxide Nanoparticles, Superparamagnetic Iron Oxide Nanoparticles, and Gold Nanoparticles. Inorganic nanoparticles have been currently investigated as contrast agents in clinical practice [130]. Iron oxide nanoparticles and superparamagnetic iron oxide nanoparticles have been studied extensively as contrast agents because they enhance the negative contrasts and give us darker images of the interest regions in magnetic resonance imaging (MRI). Iron oxide nanoparticles have a magnetic moment to be changed by an ambient thermal energy. This system has been widely used for MRI contrast enhancements, as well as tissuespecific release of therapeutic agents [131]. Superparamagnetic iron oxide (SPIO) nanoparticles are either form which includes the inside of the core of magnetic nanoparticles with a polymeric coating or a homogeneous integration into the polymeric nanoparticles [4]. This nanoparticular system has a small size with the range of 3-6 $\mathrm{nm}$ of the core size and $20-150 \mathrm{~nm}$ after dextran coating such as Feridex and Combidex, which is a superior biocompatible magnetic material-based biomedical technique with respect to other magnetic materials, both based on oxides or pure metals [132]. In addition, this system can deliver anticancer drugs such as doxorubicin and methotrexate, which is used as theranostic cargo system due to its small size $[133,134]$. In addition, the gold nanoparticles were used together with the iron oxide nanoparticles to study the MRI contrast agents, as well as the optical probes exploiting the reflectance signal of the gold nanoparticles [135].

The gold nanoparticles can also be used in an ultrasensitive assay technique to detect cancers [136]. Peng et al. introduced the functionalized gold nanoparticles in combination with the pattern recognition methods to diagnose the lung cancer from breath testing [137]. This method is an in vitro sensor array technique of the detection of biomarkers in exhaled breath of lung cancer patients as a noninvasive diagnostic tool. In addition, Thaxton et al. reported the combination system based on the magnetic microparticles and the gold nanoparticles conjugated with the prostate-specific antigen- (PSA-) specific antibodies to diagnose prostate cancers [138]. In this system, the magnetic microparticles conjugated with PSA-specific antibodies were used to extract the traceable amounts of PSA in the serum samples from patients, and the gold nanoparticles with PSAspecific antibodies and short DNA sequences (the barcodes) were attached to detect this analyte for in vitro barcode assay.

4.5. Carbon-Based Nanoplatforms-Carbon Nanotubes and Graphene. Graphite is one of the carbon-based natural materials that are widely used in large-scale industrial applications such as steelmaking and battery electrodes [139]. From graphite, carbon-based nanobiomaterials have been engineered with the deeper nanofabrication techniques, 
which include carbon nanotubes and graphene [140]. These nanobiomaterials are currently and widely regarded as highly attractive biomedical application systems that have a multifunctional nature. In addition, they are incorporated into the conventional existing nanobiomaterials, so-called the hybrid system with further function [141, 142].

Carbon nanotubes are cylindrical nanostructure-based carbon materials, which are synthesized by an arc discharge or chemical vapor deposition of graphite [143]. They are shaped like rolling sheets of carbon into the hollow tubes. Their sizes are ultrasmall, 0.4 to $2 \mathrm{~nm}$ in diameter of singlewalled carbon nanotubes (SWCNTs) [144, 145]. This system can also be modified to be suitable for biological applications with an addition of functional groups, targeting molecules, and polymers, and so forth to enhance the solubility and biocompatibility [146]. Ruggiero et al. studied the biodistribution and glomerular filtration in the kidney of SWCNT with fluorescence label. This system was applied to the near infrared (NIR) fluorescence imaging and dynamic positron emission tomography (PET) imaging [147]. For the enhancement of solubility of the carbon nanotubes, Liu et al. reported the water soluble carbon nanotubes functionalized with PEG [148]. In addition, this water soluble carbon nanotube system with radio labels and RGD peptide targeted integrins $\alpha_{v} \beta_{3}$ for the treatment of human glioblastoma and human colorectal cancer in the U87MG and HT-29 tumor xenograft models. They checked the biodistribution of this system using PET scan in the murine tumor model after intravenous injection.

Graphene is a single planar sheet structured carbonbased material, which is a single atomic plane of graphite, in a honey comb crystal lattice [149]. It is isolated by a simple method for extracting graphene from graphite via exfoliation [150]. Graphene is similar to carbon nanotubes in that it has similar electrical, optical, and thermal properties, although the structure of graphene is twodimensional atomic sheet different from carbon nanotubes [151]. Graphene can be an attractive material since it has the possibility of being engineered to be structurally thin and flexible. For the biomedical applications, graphene oxide and reduced graphene oxide are more commonly used due to its solubility in aqueous environments and capability of chemical functionalization [152]. Graphene oxide was produced by the oxidation of graphite under acidic conditions (e.g., the modified Hummer's method), and reduced graphene oxide was provided from a reduction of graphene oxide with several reducing reagents (e.g., hydrazine) [153]. They are applied to the biomedical nanomedicine including injectable drug delivery systems for anticancer therapy as carbon nanotubes [154]. For example, the nanographene sheet (NGS) for photothermal therapy (PTT) was reported by Shi et al. [155], which was a six-armed PEG-NGS of $10-50 \mathrm{~nm}$ in size with fluorescence labeling for PTT of cancers in the models of $4 \mathrm{~T} 1$ bearing Balb/c mice, as well as $\mathrm{KB}$ and U87MG xenograft models after intravenous injection. In addition, reduced graphene oxide was applied to PTT in tumor models by Yang et al. [156] who used the reduced graphene oxide conjugated with the chimeric form of anti-CD105 mAb, TRC 105, to target and detect the tumor vasculature in the living mice as a theranostic agent.

4.6. Dendrimers. Dendrimers are three-dimensional spherical-shaped nanobiomaterials with repeated branches of dendron which contains a single chemical group, called a focal point $[157,158]$. The structure of dendrimers consists of a core of initiator, repeated branching units, terminal functional groups, and void spaces, which are rooms for molecular cargo [159]. In the globular and nanosized structures of dendrimers, the terminal functional groups of the outer surface are essential in determining the properties of dendritic macromolecules, which can be interacted and conjugated with other molecules to target the cancer cells and tissues. The commonly used dendrimers in nanomedicines are polyamidoamines (PAMAM) [160], poly(L-lysine) scaffold dendrimers (PLL) [161], polyesters (PGLSA-OH) [162], polypropylimines (PPI) [163], and poly(2,2-bis(hydroxymethyl) propionic acid scaffold dendrimers (bis-MPA) [164]. Some of them are commercially available such as PAMAM dendrimers (Starburst) and PPI dendrimers (Astramol) $[165,166]$. Dendrimers can be applied to cancer targeting conjugated with the targeting ligands such as folate, transferrin, antibodies, peptides, and aptamers [167]. In addition, multifunctionality of dendrimers can be a major advantage based on an incorporation of anticancer therapeutics as well as imaging agents $[158,165,168]$.

4.7. Virus-Based Nanoplatforms (Phage System). Virus-based nanomaterials have been dramatically investigated in recent years [169]. Viruses are biochemical complexes composed of genomic and proteomic materials. The desired functions of materials for bionanomedicine can be engineered by designing the shape and size of nanoparticles as well as the specific sequence of DNA and proteins. The self-assembled viral architecture can occur in a wide range of shapes and sizes [170] and can offer remarkable structural features of virus that make them excellent candidates for bionanomedicine $[171,172]$. Advancements in nanoscale biological engineering of viral particles provide a development of novel pathways to develop nanomedicine for cancer therapeutics. Various types of viruses, such as adenovirus, adeno-associated virus, and bacteriophages, have been utilized for targeted cancer therapy and imaging through genetic and chemical modifications of the virus [173-175].

A bacteriophage (phage) is a prokaryotic virus that can infect the bacterial host cells exploiting the host's biosynthetic machinery to produce many identical copies of the phage itself [176]. There are many types of phages with different genomic materials, replication processes, and shapes such as linear (M13, Fd, and F1) [177] or spherical (MS2) [178]. Some shapes are quite sophisticated; for example, T4 and T7 phages possess an icosahedral head and a long tail connected through a cylindrical body [170]. Over the last two decades, the biochemical landscape of the phage structure has been greatly expanded through genetic engineering [179-182] and site-specific organic synthesis approaches [183-186]. Through 
genetic engineering, many foreign or synthetic DNAs have been integrated into the phage genome and expressed at various sites of the phage body [182, 187]. Several groups are investigating the engineered filamentous bacteriophage for in vivo screening via phage display within organs [188] or cancerous tissue $[189,190]$, for the purpose of targeted drug delivery [191, 192] or as an imaging agent [193]. For gene delivery applications, therapeutic genetic material can be incorporated into the phage DNA and carried into the cells following a receptor uptake [194]. The phage can be locally targeted to the cell receptors by incorporation of specific targeting and/or internalization peptides (i.e., via RGD or other ligands). To make the phage even more effective than the DNA delivery vehicles, phage can be further decorated with peptides that facilitate endosomal escape or nuclear localization motifs that target the nuclear envelope [195]. The most widely used bacteriophages for gene delivery are M13 filamentous phages [173, 196, 197] and lambda phages [198]. To enhance gene delivery efficiency, the phage with the multifunctional peptides can be produced using a phagemid system, which facilitates manipulation of expressed proteins on viral vectors [197]. Phage display technology has allowed for identification of novel homing peptides that target the unknown cell surface proteins. The targeting peptides can be incorporated into the phage coat proteins through genetic engineering techniques or chemical modifications to improve targeting efficiency [194]. These include peptides (RGD, glioma-binding peptide) [197, 199], HER2 receptor targeting antibody [173], growth factors (EGF and FGF2) [200, 201], and the penton base of adenovirus [198]. Hajitou et al. constructed a hybrid phage with two genes from the phage and nucleus integrating gene from an adeno-associated virus (AAV), called inverted terminal repeats [194]. Although eukaryotic viruses such as AAV have fantastic transgene delivery capabilities, they require an elimination of the native tropism for mammalian cells. In contrast, M13 phages have no tropism for mammalian cells; however, their gene delivery efficiency is poor. Thus, there has been an effort to combine the advantageous aspects of AAV and M13 phages into a single system [194]. This phage displayed integrin-binding peptides (cyclic RGD) on the minor coat proteins and carried the herpes simplex virus thymidine kinase gene (HSVtk). The resulting AAV/phage system provided a superior tumor transduction over the phage alone and was used as the PET imaging agent with $\left[{ }^{18} \mathrm{~F}\right]$ FDG and $\left[{ }^{18} \mathrm{~F}\right] \mathrm{FEAU}$ as well as cancer therapeutics with ganciclovir treatment (Table 1). Ghosh et al. investigated the selective tumor targeting phage-based material for in vivo imaging for prostate cancer, acidic and rich in cysteine (SPARC), which is upregulated in various cancers and correlated with poor prognosis [202]. The capsid organization of M13 spatially separates the targeting and imaging moieties. Using p3 to display the targeting ligands (SPARC-binding peptide, SPPTGIN) while assembling multiple magnetic iron oxide nanoparticles (MNPs) along the p8 capsid achieves effective targeting and delivers a larger payload of MNPs per SPARC compared with directly functionalized nanoparticles (Table 1). Compared with nanoparticles that are directly functionalized with targeting peptides, this approach improves the contrast because each SPARC-targeting molecule delivers a larger number of nanoparticles into the cells. Moreover, the targeting ligand and nanoparticles could be easily exchanged for others, making this platform attractive for in vivo screening and molecular detection (Table 1).

\section{Applications of Targeted Delivery Systems from Cells to Clinics}

Nanoplatforms generally have the potential to be applied as cancer diagnosis, imaging, and treatment in vitro and in vivo. Targeted delivery strategies of nanoplatforms are special formulations and carriers with anticancer drugs such as pegylated liposomes, polymeric nanoparticles/micelles, and albumin-based drug carriers. These nanoplatforms that we mentioned above can be applicable to the biomedical approaches such as cancer diagnostics [145], anticancer therapy [203], nanoimaging [204], bimodal imaging [205, 206], and real-time intraoperative imaging [26]. The FDA has approved clinical use of a significant number of anticancer drug products in the nanometer size range including the applications in Table 3. In particular, nanoimaging has become based on the diagnostic potential of an earlier detection in the cancer and other human diseases [207]. In the case of bimodal contrast imaging, bimodal contrast agents allow the assessment of regions of interest using two independent imaging modalities such as MRI reagents and fluorescent agents. In the case of MRI and fluorescence imaging, MRI has an excellent spatial resolution, and fluorescence imaging compensates for the sensitivity of MRI overcoming the limitations of a single-modality imaging [208]. For intraoperative fluorescence imaging, the first human trial in advanced-stage ovarian cancer proceeded with tumorspecific folate receptor- $\alpha$ targeted fluorescent agent [26]. This study offers the potential application of intraoperative staging with tumor-specific fluorescence imaging in patients with ovarian cancer by folate receptor- $\alpha$ overexpression.

\section{Conclusion}

Human genomic map has accelerated the current biomedical application for the improvement of human healthcare and future therapies encompassing a full understanding of the gene function. Increasing information regarding the gene regulation process will provide the fundamental knowledge for the development of novel therapy in the disease state of cancer. For this streamline of biomedical research, nanoplatforms can take the center stage of participating in the development of targeted nanomedicine for anticancer therapy in the foreseeable future. Using these nanoplatform techniques, targeted anticancer therapy of nanomedicine without toxicity will be able to detect, confirm, and treat various types of cancers as a part of personalized medicine. This therapeutic potential will require more approaches to develop ideal targeted nanoplatforms overcoming toxicity and enhancing biocompatibility, as well as multifunctionality 
of nanoplatforms. We believe that these cancer targeting studies of nanoplatforms can contribute to the scientific achievement of nanotechnology and nanoplatforms for the development of targeted anticancer therapeutics.

\section{Conflict of Interests}

The authors declare that there is no conflict of interests regarding the publication of this paper.

\section{Acknowledgments}

This work is supported by Korean Health Technology R\&D Project (A120266), Ministry of Health and Welfare, the National Research Foundation of Korea (NRF) funded by the Ministry of Education, Science and Technology (NRF2012-0002988, 2012R1A2A2A01045602), and Inha University grant.

\section{References}

[1] American Cancer Society, Cancer Facts and Figures 2013, American Cancer Society, Atlanta, Ga, USA, 2013.

[2] S. Svenson, "Clinical translation of nanomedicines," Current Opinion in Solid State and Materials Science, vol. 16, no. 6, pp. 287-294, 2012.

[3] A. M. Mohs and J. M. Provenzale, "Applications of nanotechnology to imaging and therapy of brain tumors," Neuroimaging Clinics of North America, vol. 20, no. 3, pp. 283-292, 2010.

[4] Y.-E. L. Koo, G. R. Reddy, M. Bhojani et al., "Brain cancer diagnosis and therapy with nanoplatforms," Advanced Drug Delivery Reviews, vol. 58, no. 14, pp. 1556-1577, 2006.

[5] National Nanotechnology Initiative (NNI), "What is nanotechnology?" http://www.nano.gov/.

[6] H. S. Choi and J. V. Frangioni, "Nanoparticles for biomedical imaging: fundamentals of clinical translation," Molecular Imaging, vol. 9, no. 6, pp. 291-310, 2010.

[7] J. Shi, A. R. Votruba, O. C. Farokhzad, and R. Langer, "Nanotechnology in drug delivery and tissue engineering: from discovery to applications," Nano Letters, vol. 10, no. 9, pp. 32233230, 2010.

[8] L. Zhang, F. X. Gu, J. M. Chan, A. Z. Wang, R. S. Langer, and O. C. Farokhzad, "Nanoparticles in medicine: therapeutic applications and developments," Clinical Pharmacology and Therapeutics, vol. 83, no. 5, pp. 761-769, 2007.

[9] T. Lammers, W. E. Hennink, and G. Storm, "Tumour-targeted nanomedicines: principles and practice," British Journal of Cancer, vol. 99, no. 3, pp. 392-397, 2008.

[10] T. Lammers, F. Kiessling, W. E. Hennink, and G. Storm, "Drug targeting to tumors: principles, pitfalls and (pre-) clinical progress," Journal of Controlled Release, vol. 161, no. 2, pp. 175$187,2012$.

[11] D. W. Northfelt, B. J. Dezube, J. A. Thommes et al., "Pegylatedliposomal doxorubicin versus doxorubicin, bleomycin, and vincristine in the treatment of AIDS-related Kaposi's sarcoma: results of a randomized phase III clinical trial," Journal of Clinical Oncology, vol. 16, no. 7, pp. 2445-2451, 1998.

[12] R. Strother and D. Matei, "Pegylated liposomal doxorubicin in ovarian cancer," Therapeutics and Clinical Risk Management, vol. 5, no. 3, pp. 639-650, 2009.
[13] A. D. Bangham, M. M. Standish, and J. C. Watkins, "Diffusion of univalent ions across the lamellae of swollen phospholipids," Journal of Molecular Biology, vol. 13, no. 1, pp. 238-252, 1965.

[14] K. Sen and M. Mandal, "Second generation liposomal cancer therapeutics: transition from laboratory to clinic," International Journal of Pharmaceutics, vol. 448, no. 1, pp. 28-43, 2013.

[15] H. Maeda, H. Nakamura, and J. Fang, "The EPR effect for macromolecular drug delivery to solid tumors: improvement of tumor uptake, lowering of systemic toxicity, and distinct tumor imaging in vivo," Advanced Drug Delivery Reviews, vol. 65, no. 1, pp. 71-79, 2013.

[16] O. C. Farokhzad and R. Langer, "Impact of nanotechnology on drug delivery," ACS Nano, vol. 3, no. 1, pp. 16-20, 2009.

[17] M. Zhao, M. Yang, X.-M. Li et al., “Tumor-targeting bacterial therapy with amino acid auxotrophs of GFP-expressing Salmonella typhimurium," Proceedings of the National Academy of Sciences of the United States of America, vol. 102, no. 3, pp. 755-760, 2005.

[18] N. T. Kimura, S. Taniguchi, K. Aoki, and T. Baba, "Selective localization and growth of Bifidobacterium bifidum in mouse tumors following intravenous administration," Cancer Research, vol. 40, no. 6, pp. 2061-2068, 1980.

[19] Y. Matsumura and H. Maeda, "A new concept for macromolecular therapeutics in cancer chemotherapy: mechanism of tumoritropic accumulation of proteins and the antitumor agent smancs," Cancer Research, vol. 46, no. 12, part 1, pp. 6387-6392, 1986.

[20] J. Fang, H. Nakamura, and H. Maeda, “The EPR effect: unique features of tumor blood vessels for drug delivery, factors involved, and limitations and augmentation of the effect," Advanced Drug Delivery Reviews, vol. 63, no. 3, pp. 136-151, 2011.

[21] H. Gelderblom, J. Verweij, K. Nooter, and A. Sparreboom, "Cremophor EL: the drawbacks and advantages of vehicle selection for drug formulation," European Journal of Cancer, vol. 37, no. 13, pp. 1590-1598, 2001.

[22] T. M. Allen and P. R. Cullis, "Drug delivery systems: entering the mainstream," Science, vol. 303, no. 5665, pp. 1818-1822, 2004.

[23] D. Peer, J. M. Karp, S. Hong, O. C. Farokhzad, R. Margalit, and R. Langer, "Nanocarriers as an emerging platform for cancer therapy," Nature Nanotechnology, vol. 2, no. 12, pp. 751-760, 2007.

[24] M. E. Davis, Z. Chen, and D. M. Shin, "Nanoparticle therapeutics: an emerging treatment modality for cancer," Nature Reviews Drug Discovery, vol. 7, no. 9, pp. 771-782, 2008.

[25] R. K. Jain and T. Stylianopoulos, "Delivering nanomedicine to solid tumors," Nature Reviews Clinical Oncology, vol. 7, no. 11, pp. 653-664, 2010.

[26] G. M. Van Dam, G. Themelis, L. M. A. Crane et al., "Intraoperative tumor-specific fluorescence imaging in ovarian cancer by folate receptor- $\alpha$ targeting: first in-human results," Nature Medicine, vol. 17, no. 10, pp. 1315-1319, 2011.

[27] L. W. Seymour, D. R. Ferry, D. Anderson et al., "Hepatic drug targeting: phase I evaluation of polymer-bound doxorubicin," Journal of Clinical Oncology, vol. 20, no. 6, pp. 1668-1676, 2002.

[28] G. D. Lewis Phillips, G. Li, D. L. Dugger et al., "Targeting HER2positive breast cancer with trastuzumab-DM1, an antibodycytotoxic drug conjugate," Cancer Research, vol. 68, no. 22, pp. 9280-9290, 2008.

[29] Z. M. Qian, H. Li, H. Sun, and K. Ho, “Targeted drug delivery via the transferrin receptor-mediated endocytosis pathway," Pharmacological Reviews, vol. 54, no. 4, pp. 561-587, 2002. 
[30] B. Gupta, T. S. Levchenko, and V. P. Torchilin, "Intracellular delivery of large molecules and small particles by cellpenetrating proteins and peptides," Advanced Drug Delivery Reviews, vol. 57, no. 4, pp. 637-651, 2005.

[31] D. Neri and R. Bicknell, "Tumour vascular targeting," Nature Reviews Cancer, vol. 5, no. 6, pp. 436-446, 2005.

[32] K. Temming, R. M. Schiffelers, G. Molema, and R. J. Kok, "RGD-based strategies for selective delivery of therapeutics and imaging agents to the tumour vasculature," Drug Resistance Updates, vol. 8, no. 6, pp. 381-402, 2005.

[33] R. Pasqualini, E. Koivunen, R. Kain et al., "Aminopeptidase N is a receptor for tumor-homing peptides and a target for inhibiting angiogenesis," Cancer Research, vol. 60, no. 3, pp. 722-727, 2000.

[34] Y. Matsumura and K. Kataoka, "Preclinical and clinical studies of anticancer agent-incorporating polymer micelles," Cancer Science, vol. 100, no. 4, pp. 572-579, 2009.

[35] S. Nie, "Editorial: understanding and overcoming major barriers in cancer nanomedicine," Nanomedicine, vol. 5, no. 4, pp. 523-528, 2010.

[36] F. Perche and V. P. Torchilin, "Recent trends in multifunctional liposomal nanocarriers for enhanced tumor targeting," Journal of Drug Delivery, vol. 2013, Article ID 705265, 32 pages, 2013.

[37] A. Srivastavaa, I. B. O. 'Connora, A. Panditb, and J. G. Wall, "Polymer-antibody fragment conjugates for biomedical applications," Progress in Polymer Science, 2013.

[38] P. E. Kintzel and R. T. Dorr, "Anticancer drug renal toxicity and elimination: dosing guidelines for altered renal function," Cancer Treatment Reviews, vol. 21, no. 1, pp. 33-64, 1995.

[39] A. Remesh, "Toxicities of anticancer drugs and its management," International Journal of Basic \& Clinical Pharmacology, vol. 1, no. 1, pp. 2-12, 2012.

[40] R. K. Jain and T. Stylianopoulos, "Delivering nanomedicine to solid tumors," Nature Reviews Clinical Oncology, vol. 7, no. 11, pp. 653-664, 2010.

[41] N. Kamaly, Z. Xiao, P. M. Valencia, A. F. Radovic-Moreno, and O. C. Farokhzad, "Targeted polymeric therapeutic nanoparticles: design, development and clinical translation," Chemical Society Reviews, vol. 41, no. 7, pp. 2971-3010, 2012.

[42] D. C. Julien, S. Behnke, G. Wang, G. K. Murdoch, and R. A. Hill, "Utilization of monoclonal antibody-targeted nanomaterials in the treatment of cancer," $m A b s$, vol. 3, no. 5, pp. 467-478, 2011.

[43] J. Kopeček, "Polymer-drug conjugates: origins, progress to date and future directions," Advanced Drug Delivery Reviews, vol. 65, no. 1, pp. 49-59, 2013.

[44] A. Accardo, D. Tesauro, and G. Morelli, "Peptide-based targeting strategies for simultaneous imaging and therapy with nanovectors," Polymer Journal , vol. 45, no. 5, pp. 481-493, 2013.

[45] S. Sofou, "Surface-active liposomes for targeted cancer therapy," Nanomedicine, vol. 2, no. 5, pp. 711-724, 2007.

[46] M. Wang and M. Thanou, "Targeting nanoparticles to cancer," Pharmacological Research, vol. 62, no. 2, pp. 90-99, 2010.

[47] O. Tacar, P. Sriamornsak, and C. R. Dass, "Doxorubicin: an update on anticancer molecular action, toxicity and novel drug delivery systems," Journal of Pharmacy and Pharmacology, vol. 65, no. 2, pp. 157-170, 2013.

[48] M. Volkova and R. Russell III, "Anthracycline cardiotoxicity: prevalence, pathogenesis and treatment," Current Cardiology Reviews, vol. 7, no. 4, pp. 214-220, 2011.

[49] V. Krishnan and A. K. Rajasekaran, "Clinical nanomedicine: a solution to the chemotherapy conundrum in pediatric leukemia therapy," Clinical Pharmacology and Therapeutics, 2013.
[50] R. van der Meela, L. J. C. Vehmeijera, R. J. Koka, G. Storma, and E. V. B. van Gaala, "Ligand-targeted particulate nanomedicines undergoing clinical evaluation: current status," Advanced Drug Delivery Reviews, vol. 65, no. 10, pp. 1284-1298, 2013.

[51] D. Y. Alakhova, Y. Zhao, S. Li, and A. V. Kabanov, "Effect of doxorubicin/pluronic SP1049C on tumorigenicity, aggressiveness, DNA methylation and stem cell markers in murine leukemia," PloS ONE, vol. 8, no. 8, Article ID e72238, 2013.

[52] Y. Matsumura, T. Hamaguchi, T. Ura et al., "Phase I clinical trial and pharmacokinetic evaluation of NK911, a micelleencapsulated doxorubicin," British Journal of Cancer, vol. 91, no. 10, pp. 1775-1781, 2004.

[53] P. Cozzi, N. Mongelli, and A. Suarato, "Recent anticancer cytotoxic agents," Current Medicinal Chemistry-Anti-Cancer Agents, vol. 4, no. 2, pp. 93-121, 2004.

[54] E. K. Rowinsky, M. Wright, B. Monsarrat, and R. C. Donehower, "Clinical pharmacology and metabolism of Taxol (paclitaxel): update 1993," Annals of Oncology, vol. 5, supplement, pp. S7-S16, 1994.

[55] E. K. Rowinsky and R. C. Donehower, "Drug therapy: paclitaxel (taxol)," The New England Journal of Medicine, vol. 332, no. 15, pp. 1004-1014, 1995.

[56] H. A. Azim Jr. and A. Awada, "Clinical development of new formulations of cytotoxics in solid tumors," Current Opinion in Oncology, vol. 24, no. 3, pp. 325-331, 2012.

[57] U. Fasol, A. Frost, M. Büchert et al., "Vascular and pharmacokinetic effects of EndoTAG-1 in patients with advanced cancer and liver metastasis," Annals of Oncology, vol. 23, no. 4, pp.10301036, 2012.

[58] S. Koudelka and J. Turanek, "Liposomal paclitaxel formulations," Journal of Controlled Release, vol. 163, no. 3, pp. 322-334, 2012.

[59] T.-Y. Kim, D.-W. Kim, J.-Y. Chung et al., "Phase I and pharmacokinetic study of Genexol-PM, a Cremophor-free, polymeric micelle-formulated paclitaxel, in patients with advanced malignancies," Clinical Cancer Research, vol. 10, no. 11, pp. 3708-3716, 2004.

[60] T. Negishi, F. Koizumi, H. Uchino et al., "NK105, a paclitaxelincorporating micellar nanoparticle, is a more potent radiosensitising agent compared to free paclitaxel," British Journal of Cancer, vol. 95, no. 5, pp. 601-606, 2006.

[61] V. L. Galic, T. J. Herzog, J. D. Wright, and S. N. Lewin, "Paclitaxel poliglumex for ovarian cancer," Expert Opinion on Investigational Drugs, vol. 20, no. 6, pp. 813-821, 2011.

[62] T. Hamaguchi, Y. Matsumura, M. Suzuki et al., "NK105, a paclitaxel-incorporating micellar nanoparticle formulation, can extend in vivo antitumour activity and reduce the neurotoxicity of paclitaxel," British Journal of Cancer, vol. 92, no. 7, pp. 12401246, 2005.

[63] X. Wang and Z. Guo, "Targeting and delivery of platinum-based anticancer drugs," Chemical Society Reviews, vol. 42, no. 1, pp. 202-2224, 2013.

[64] J. S. Butler and P. J. Sadler, “Targeted delivery of platinum-based anticancer complexes," Current Opinion in Chemical Biology, vol. 17, no. 2, pp. 175-188, 2013.

[65] W. C. Zamboni, "Concept and clinical evaluation of carriermediated anticancer agents," Oncologist, vol. 13, no. 3, pp. 248260, 2008.

[66] R. Plummer, R. H. Wilson, H. Calvert et al., "A Phase I clinical study of cisplatin-incorporated polymeric micelles (NC-6004) in patients with solid tumours," British Journal of Cancer, vol. 104, no. 4, pp. 593-598, 2011. 
[67] I. Kümler, N. Brünner, J. Stenvang, E. Balslev, and D. L. Nielsen, "A systematic review on topoisomerase 1 inhibition in the treatment of metastatic breast cancer," Breast Cancer Research and Treatment, vol. 138, no. 2, pp. 347-358, 2013.

[68] L. Zhang, Y. Hu, X. Jiang, C. Yang, W. Lu, and Y. H. Yang, "Camptothecin derivative-loaded poly(caprolactone-co-lactide)-b-PEG-b- poly(caprolactone-co-lactide) nanoparticles and their biodistribution in mice," Journal of Controlled Release, vol. 96, no. 1, pp. 135-148, 2004.

[69] W. C. Zamboni, S. Ramalingam, D. M. Friedland et al., "Phase I and pharmacokinetic study of pegylated liposomal CKD-602 in patients with advanced malignancies," Clinical Cancer Research, vol. 15, no. 4, pp. 1466-1472, 2009.

[70] C. Young, T. Schluep, J. Hwang, and S. Eliasof, "CRLX101 (formerly IT-101)-A novel nanopharmaceutical of camptothecin in clinical development," Current Bioactive Compounds, vol. 7, no. 1, pp. 8-14, 2011.

[71] T. Hamaguchi, T. Doi, T. Eguchi-Nakajima et al., "Phase I study of NK012, a novel SN-38-incorporating micellar nanoparticle, in adult patients with solid tumors," Clinical Cancer Research, vol. 16, no. 20, pp. 5058-5066, 2010.

[72] D. H. Palmer, L. S. Young, and V. Mautner, "Cancer genetherapy: clinical trials," Trends in Biotechnology, vol. 24, no. 2, pp. 76-82, 2006.

[73] L. Xu, W.-H. Tang, C.-C. Huang et al., "Systemic p53 gene therapy of cancer with immunolipoplexes targeted by antitransferrin receptor scFv," Molecular Medicine, vol. 7, no. 10, pp. 723-734, 2001.

[74] K. F. Pirollo, A. Rait, Q. Zhou et al., "Tumor-targeting nanocomplex delivery of novel tumor suppressor RB94 chemosensitizes bladder carcinoma cells in vitro and in vivo," Clinical Cancer Research, vol. 14, no. 7, pp. 2190-2198, 2008.

[75] D. W. Bartlett, H. Su, I. J. Hildebrandt, W. A. Weber, and M. E. Davis, "Impact of tumor-specific targeting on the biodistribution and efficacy of siRNA nanoparticles measured by multimodality in vivo imaging," Proceedings of the National Academy of Sciences of the United States of America, vol. 104, no. 39, pp. 15549-15554, 2007.

[76] P. P. Deshpande, S. Biswas, and V. P. Torchilin, "Current trends in the use of liposomes for tumor targeting," Nanomedicine, vol. 8, no. 9, pp. 1509-1528, 2013.

[77] W. T. Al-Jamal and K. Kostarelos, "Liposomes: from a clinically established drug delivery system to a nanoparticle platform for theranostic nanomedicine," Accounts of Chemical Research, vol. 44, no. 10, pp. 1094-1104, 2011.

[78] G. Gregoriadis, "The carrier potential of liposomes in biology and medicine. II," The New England Journal of Medicine, vol. 295, no. 14, pp. 765-770, 1976.

[79] J. L. Arias, "Liposomes in drug delivery: a patent review (2007present)," Expert Opinion on Therapeutic Patents, vol. 23, no. 11, pp. 1399-1414, 2013.

[80] M. R. Preiss and G. D. Bothun, "Stimuli-responsive liposomenanoparticle assemblies," Expert Opinion on Drug Delivery, vol. 8, no. 8, pp. 1025-1040, 2011.

[81] A. S. Manjappa, K. R. Chaudhari, M. P. Venkataraju et al., "Antibody derivatization and conjugation strategies: application in preparation of stealth immunoliposome to target chemotherapeutics to tumor," Journal of Controlled Release, vol. 150, no. 1, pp. 2-22, 2011.

[82] Y. Zhao, S. Zhang, S. Cui, B. Wang, and S. Zhang, "Peptidebased cationic liposome-mediated gene delivery," Expert Opinion on Drug Delivery, vol. 9, no. 1, pp. 127-139, 2012.
[83] M. L. Immordino, F. Dosio, and L. Cattel, "Stealth liposomes: review of the basic science, rationale, and clinical applications, existing and potential," International Journal of Nanomedicine, vol. 1, no. 3, pp. 297-315, 2006.

[84] N. K. Mehra, V. Mishra, and N. K. Jain, "Receptor-based targeting of therapeutics," Therapeutic Delivery, vol. 4, no. 3, pp. 369-394, 2013.

[85] M. Amin, A. Badiee, and M. R. Jaafari, "Improvement of pharmacokinetic and antitumor activity of PEGylated liposomal doxorubicin by targeting with $\mathrm{N}$-methylated cyclic RGD peptide in mice bearing C-26 colon carcinomas," International Journal of Pharmaceutics, vol. 458, no. 2, pp. 324-333.

[86] X. Liu, S. Ma, C. Dai et al., "Antiproliferative, antiinvasive, and proapoptotic activity of folate receptor alpha-targeted liposomal doxorubicin in nonfunctional pituitary adenoma cells," Endocrinology, vol. 154, no. 4, pp. 1414-1423, 2013.

[87] B. Xiang, D.-W. Dong, N.-Q. Shi et al., "PSA-responsive and PSMA-mediated multifunctional liposomes for targeted therapy of prostate cancer," Biomaterials, vol. 34, no. 28, pp. 69766991, 2013.

[88] ClinicalTrials.gov, http://clinicaltrials.gov/.

[89] C. Brignole, D. Marimpietri, G. Pagnan et al., "Neuroblastoma targeting by c-myb-selective antisense oligonucleotides entrapped in anti-GD2 immunoliposome: immune cell-mediated anti-tumor activities," Cancer Letters, vol. 228, no. 1-2, pp. 181186, 2005.

[90] F. Moret, D. Scheglmann, and E. Reddi, "Folate-targeted PEGylated liposomes improve the selectivity of PDT with meta-tetra(hydroxyphenyl)chlorin (m-THPC)," Photochemical \& Photobiological Sciences, vol. 12, no. 5, pp. 823-834, 2013.

[91] Z. Zhang and J. Yao, "Preparation of irinotecan-loaded folatetargeted liposome for tumor targeting delivery and its antitumor activity," AAPS PharmSciTech, vol. 13, no. 3, pp. 802-810, 2012.

[92] K. F. Yu, W. Q. Zhang, L. M. Luo et al., "The antitumor activity of a doxorubicin loaded, iRGD-modified sterically-stabilized liposome on B16-F10 melanoma cells: in vitro and in vivo evaluation," International Journal of Nanomedicine, vol. 8, pp. 2473-2485, 2013.

[93] A. Wicki, C. Rochlitz, A. Orleth et al., "Targeting tumorassociated endothelial cells: Anti-VEGFR2 immunoliposomes mediate tumor vessel disruption and inhibit tumor growth," Clinical Cancer Research, vol. 18, no. 2, pp. 454-464, 2012.

[94] C. Ma, X. Li, X. liang, K. Jin, J. Cao, and W. Xu, "Novel beta-dicarbonyl derivatives as inhibitors of aminopeptidase $\mathrm{N}$ (APN)," Bioorganic \& Medicinal Chemistry Letters, vol. 23, no. 17, pp. 4948-4952, 2013.

[95] A. H. Negussie, J. L. Miller, G. Reddy, S. K. Drake, B. J. Wood, and M. R. Dreher, "Synthesis and in vitro evaluation of cyclic NGR peptide targeted thermally sensitive liposome," Journal of Controlled Release, vol. 143, no. 2, pp. 265-273, 2010.

[96] S. Tan, X. Li, Y. Guo, and Z. Zhang, "Lipid-enveloped hybrid nanoparticles for drug delivery," Nanoscale, vol. 5, no. 3, pp. 860-872, 2013.

[97] G. Von Maltzahn, J.-H. Park, K. Y. Lin et al., "Nanoparticles that communicate in vivo to amplify tumour targeting," Nature Materials, vol. 10, no. 7, pp. 545-552, 2011.

[98] W. Huang, J. Zhang, H. C. Dorn, and C. Zhang, "Assembly of bio-nanoparticles for double controlled drug release," PLoS ONE, vol. 8, no. 9, Article ID e74679, 2013. 
[99] F. Karchemski, D. Zucker, Y. Barenholz, and O. Regev, "Carbon nanotubes-liposomes conjugate as a platform for drug delivery into cells," Journal of Controlled Release, vol. 160, no. 2, pp. 339345, 2012.

[100] A. S. Narang, R. K. Chang, and M. A. Hussain, "Pharmaceutical development and regulatory considerations for nanoparticles and nanoparticulate drug delivery systems," Journal of Pharmaceutical Sciences, vol. 102, no. 11, pp. 3867-3882, 2013.

[101] R. Duncan, "Polymer conjugates as anticancer nanomedicines," Nature Reviews Cancer, vol. 6, no. 9, pp. 688-701, 2006.

[102] S. S. Banerjee, N. Aher, R. Patil, and J. Khandare, "Poly(ethylene glycol)-prodrug conjugates: concept, design, and applications," Journal of Drug Delivery, vol. 2012, Article ID 103973, 17 pages, 2012.

[103] H. Sah, L. A. Thoma, H. R. Desu, E. Sah, and G. C. Wood, "Concepts and practices used to develop functional PLGA-based nanoparticulate systems," International Journal of Nanomedicine, vol. 8, pp. 747-765, 2013.

[104] F. Danhier, E. Ansorena, J. M. Silva, R. Coco, A. Le Breton, and V. Préat, "PLGA-based nanoparticles: an overview of biomedical applications," Journal of Controlled Release, vol. 161, no. 2, pp. 505-522, 2012.

[105] S. Acharya and S. K. Sahoo, "PLGA nanoparticles containing various anticancer agents and tumour delivery by EPR effect," Advanced Drug Delivery Reviews, vol. 63, no. 3, pp. 170-183, 2011.

[106] F. Danhier, N. Lecouturier, B. Vroman et al., "Paclitaxel-loaded PEGylated PLGA-based nanoparticles: in vitro and in vivo evaluation," Journal of Controlled Release, vol. 133, no. 1, pp. 1117, 2009.

[107] J. Liang, Y. Luo, and H. Zhao, "Synthetic biology: putting synthesis into biology," Wiley Interdisciplinary Reviews, vol. 3, no. 1, pp. 7-20, 2011.

[108] W. Hasan, K. Chu, A. Gullapalli et al., "Delivery of multiple siRNAs using lipid-coated PLGA nanoparticles for treatment of prostate cancer," Nano Letters, vol. 12, no. 1, pp. 287-292, 2012.

[109] C. Chittasupho, S.-X. Xie, A. Baoum, T. Yakovleva, T. J. Siahaan, and C. J. Berkland, "ICAM-1 targeting of doxorubicin-loaded PLGA nanoparticles to lung epithelial cells," European Journal of Pharmaceutical Sciences, vol. 37, no. 2, pp. 141-150, 2009.

[110] C. Liang, Y. Yang, Y. Ling, Y. Huang, T. Li, and X. Li, "Improved therapeutic effect of folate-decorated PLGA-PEG nanoparticles for endometrial carcinoma," Bioorganic and Medicinal Chemistry, vol. 19, no. 13, pp. 4057-4066, 2011.

[111] H. Zhao and L. Y. L. Yung, "Addition of TPGS to folateconjugated polymer micelles for selective tumor targeting," Journal of Biomedical Materials Research - Part A, vol. 91, no. 2, pp. 505-518, 2009.

[112] R. M. Taylor, V. Severns, D. C. Brown, M. Bisoffi, and L. O. Sillerud, "Prostate cancer targeting motifs: Expression of $\alpha \mathrm{v} \beta 3$, neurotensin receptor 1 , prostate specific membrane antigen, and prostate stem cell antigen in human prostate cancer cell lines and xenografts," Prostate, vol. 72, no. 5, pp. 523-532, 2012.

[113] S. Dhar, F. X. Gu, R. Langer, O. C. Farokhza, and S. J. Lippard, "Targeted delivery of cisplatin to prostate cancer cells by aptamer functionalized Pt(IV) prodrug-PLGA - PEG nanoparticles," Proceedings of the National Academy of Sciences of the United States of America, vol. 105, no. 45, pp. 17356-17361, 2008.

[114] F. Danhier, V. Pourcelle, J. Marchand-Brynaert, C. Jérôme, O. Feron, and V. Préat, "Targeting of tumor endothelium by RGDgrafted PLGA-nanoparticles," Methods in Enzymology, vol. 508, pp. 157-175, 2012.
[115] R. Hassert, P. G. Hoffmeister, M. Pagel, M. Hacker, M. SchulzSiegmund, and A. G. Beck-Sickinger, "On-resin synthesis of an acylated and fluorescence-labeled cyclic integrin ligand for modification of poly(lactic-co-glycolic acid)," Chemistry \& Biodiversity, vol. 9, no. 11, pp. 2648-2658, 2012.

[116] A. Aravind, P. Jeyamohan, R. Nair et al., "AS1411 aptamer tagged PLGA-lecithin-PEG nanoparticles for tumor cell targeting and drug delivery," Biotechnology and Bioengineering, vol.109, no. 11, pp. 2920-2931, 2012.

[117] J. Guo, X. Gao, L. Su et al., "Aptamer-functionalized PEGPLGA nanoparticles for enhanced anti-glioma drug delivery," Biomaterials, vol. 32, no. 31, pp. 8010-8020, 2011.

[118] B. Fadeel, "Clear and present danger? Engineered nanoparticles and the immune system," Swiss Medical Weekly, vol. 142, Article ID w13609, 2012.

[119] R. Becker, C. Dembek, L. A. White, and L. P. Garrison, “The cost offsets and cost-effectiveness associated with pegylated drugs: a review of the literature," Expert Review of Pharmacoeconomics \& Outcomes Research, vol. 12, no. 6, pp. 775-793, 2012.

[120] S. Kommareddy, S. B. Tiwari, and M. M. Amiji, "Longcirculating polymeric nanovectors for tumor-selective gene delivery," Technology in Cancer Research and Treatment, vol. 4, no. 6, pp. 615-625, 2005.

[121] S. Raha, T. Paunesku, and G. Woloschak, "Peptide-mediated cancer targeting of nanoconjugates," Wiley Interdisciplinary Reviews: Nanomedicine and Nanobiotechnology, vol. 3, no. 3, pp. 269-281, 2011.

[122] E. Segal and R. Satchi-Fainaro, "Design and development of polymer conjugates as anti-angiogenic agents," Advanced Drug Delivery Reviews, vol. 61, no. 13, pp. 1159-1176, 2009.

[123] Y. Xiong, W. Jiang, Y. Shen et al., "A Poly( $\gamma$, l-glutamic acid)citric acid based nanoconjugate for cisplatin delivery," Biomaterials, vol. 33, no. 29, pp. 7182-7193, 2012.

[124] X. Zhao, H. Li, and R. J. Lee, “Targeted drug delivery via folate receptors," Expert Opinion on Drug Delivery, vol. 5, no. 3, pp. 309-319, 2008.

[125] X. B. Zhao and R. J. Lee, “Tumor-selective targeted delivery of genes and antisense oligodeoxyribonucleotides via the folate receptor," Advanced Drug Delivery Reviews, vol. 56, no. 8, pp. 1193-1204, 2004.

[126] L. Teng, J. Xie, and R. J. Lee, "Clinical translation of folate receptor-targeted therapeutics," Expert Opinion on Drug Delivery, vol. 9, no. 8, pp. 901-908, 2012.

[127] G. L. Zwicke, G. A. Mansoori, and C. J. Jeffery, "Utilizing the folate receptor for active targeting of cancer nanotherapeutics," Nano Reviews, vol. 3, pp. 18496-18506, 2012.

[128] X. Ming, K. Carver, and L. Wu, "Albumin-based nanoconjugates for targeted delivery of therapeutic oligonucleotides," Biomaterials, vol. 34, no. 32, pp. 7939-7949, 2013.

[129] R. K. Mittapalli, X. Liu, C. E. Adkins et al., "Paclitaxelhyaluronic nanoconjugates prolong overall survival in a preclinical brain metastases of breast cancer model," Molecular Cancer Therapeutics, vol. 12, no. 11, pp. 2389-2399, 2013.

[130] C. Boyer, M. R. Whittaker, V. Bulmus, J. Liu, and T. P. Davis, "The design and utility of polymer-stabilized ironoxide nanoparticles for nanomedicine applications," NPG Asia Materials, vol. 2, no. 1, pp. 23-30, 2010.

[131] B. Polyak and G. Friedman, "Magnetic targeting for site-specific drug delivery: Applications and clinical potential," Expert Opinion on Drug Delivery, vol. 6, no. 1, pp. 53-70, 2009. 
[132] J. Meng, J. Fan, G. Galiana et al., "LHRH-functionalized superparamagnetic iron oxide nanoparticles for breast cancer targeting and contrast enhancement in MRI," Materials Science and Engineering C, vol. 29, no. 4, pp. 1467-1479, 2009.

[133] M. K. Yu, Y. Y. Jeong, J. Park et al., "Drug-loaded superparamagnetic iron oxide nanoparticles for combined cancer imaging and therapy in vivo," Angewandte Chemie - International Edition, vol. 47, no. 29, pp. 5362-5365, 2008.

[134] N. Kohler, C. Sun, J. Wang, and M. Zhang, "Methotrexatemodified superparamagnetic nanoparticles and their intracellular uptake into human cancer cells," Langmuir, vol. 21, no. 19, pp. 8858-8864, 2005.

[135] A. Figuerola, R. Di Corato, L. Manna, and T. Pellegrino, "From iron oxide nanoparticles towards advanced iron-based inorganic materials designed for biomedical applications," Pharmacological Research, vol. 62, no. 2, pp. 126-143, 2010.

[136] G. Shipp, "Ultrasensitive measurement of protein and nucleic acid biomarkers for earlier disease detection and more effective therapies," Biotechnology Healthcare Journal, vol. 3, no. 2, pp. 3540, 2006.

[137] G. Peng, U. Tisch, O. Adams et al., "Diagnosing lung cancer in exhaled breath using gold nanoparticles," Nature Nanotechnology, vol. 4, no. 10, pp. 669-673, 2009.

[138] C. S. Thaxton, R. Elghanian, A. D. Thomas et al., "Nanoparticlebased bio-barcode assay redefines "undetectable" PSA and biochemical recurrence after radical prostatectomy," Proceedings of the National Academy of Sciences of the United States of America, vol. 106, no. 44, pp. 18437-18442, 2009.

[139] V. Singh, D. Joung, L. Zhai, S. Das, S. I. Khondaker, and S. Seal, "Graphene based materials: past, present and future," Progress in Materials Science, vol. 56, no. 8, pp. 1178-1271, 2011.

[140] C. Cha, S. R. Shin, N. Annabi, M. R. Dokmeci, and A. Khademhosseini, "Carbon-based nanomaterials: multifunctional materials for biomedical engineering," ACS Nano, vol. 7, no. 4, pp. 2891-2897, 2013.

[141] A. Biswas, I. S. Bayer, A. S. Biris, T. Wang, E. Dervishi, and F. Faupel, "Advances in top-down and bottom-up surface nanofabrication: techniques, applications \& future prospects," Advances in Colloid and Interface Science, vol. 170, no. 1-2, pp. 2-27, 2012.

[142] P. Sharma, V. Bhalla, E. S. Prasad, V. Dravid, G. Shekhawat, and C. R. Suri, "Enhancing graphene/CNT based electrochemical detection using magneto-nanobioprobes," Scientific Reports, vol. 2, pp. 877-883, 2012.

[143] K. Mallick and A. M. Strydom, "Biophilic carbon nanotubes," Colloids and Surfaces B, vol. 105, pp. 310-318, 2013.

[144] B. Kateb, M. Van Handel, L. Zhang, M. J. Bronikowski, H. Manohara, and B. Badie, "Internalization of MWCNTs by microglia: possible application in immunotherapy of brain tumors," NeuroImage, vol. 37, no. 1, pp. S9-S17, 2007.

[145] B. Kateb, K. Chiu, K. L. Black et al., "Nanoplatforms for constructing new approaches to cancer treatment, imaging, and drug delivery: What should be the policy?" NeuroImage, vol. 54, supplement 1, pp. S106-S124, 2011.

[146] A. Bianco, K. Kostarelos, C. D. Partidos, and M. Prato, "Biomedical applications of functionalised carbon nanotubes," Chemical Communications, no. 5, pp. 571-577, 2005.

[147] A. Ruggiero, C. H. Villa, E. Bander et al., "Paradoxical glomerular filtration of carbon nanotubes," Proceedings of the National Academy of Sciences of the United States of America, vol. 107, no. 27, pp. 12369-12374, 2010.
[148] Z. Liu, W. Cai, L. He et al., "in vivo biodistribution and highly efficient tumour targeting of carbon nanotubes in mice," Nature Nanotechnology, vol. 2, no. 1, pp. 47-52, 2007.

[149] C. Chung, Y.-K. Kim, D. Shin, S.-R. Ryoo, B. H. Hong, and D.-H. Min, "Biomedical applications of graphene and graphene oxide," Accounts of Chemical Research, vol. 46, no. 10, pp. 22112224, 2013.

[150] M. Cai, D. Thorpe, D. H. Adamsonb, and H. C. Schniepp, "Methods of graphite exfoliation," Journal of Materials Chemistry, vol. 22, no. 48, pp. 24992-25002, 2012.

[151] S. Z. Butler, S. M. Hollen, L. Cao et al., "Progress, challenges, and opportunities in two-dimensional materials beyond graphene," ACS Nano, vol. 7, no. 4, pp. 2898-2926, 2013.

[152] H. Shen, L. Zhang, M. Liu, and Z. Zhang, "Biomedical applications of graphene," Theranostics, vol. 2, no. 3, pp. 283-294, 2012.

[153] D. R. Dreyer, S. Park, C. W. Bielawski, and R. S. Ruoff, "The chemistry of graphene oxide," Chemical Society Reviews, vol. 39, no. 1, pp. 228-240, 2010.

[154] K. Yang, L. Feng, X. Shi, and Z. Liu, "Nano-graphene in biomedicine: theranostic applications," Chemical Society Reviews, vol. 42, no. 2, pp. 530-547, 2013.

[155] S. Shi, K. Yang, H. Hong et al., "Tumor vasculature targeting and imaging in living mice with reduced graphene oxide," Biomaterials, vol. 34, no. 12, pp. 3002-3009, 2013.

[156] K. Yang, S. Zhang, G. Zhang, X. Sun, S.-T. Lee, and Z. Liu, "Graphene in mice: ultrahigh in vivo tumor uptake and efficient photothermal therapy," Nano Letters, vol. 10, no. 9, pp. 33183323, 2010.

[157] S. Svenson and D. A. Tomalia, "Dendrimers in biomedical applications: reflections on the field," Advanced Drug Delivery Reviews, vol. 57, no. 15, pp. 2106-2129, 2005.

[158] A. R. Menjoge, R. M. Kannan, and D. A. Tomalia, "Dendrimerbased drug and imaging conjugates: design considerations for nanomedical applications," Drug Discovery Today, vol. 15, no. 56, pp. 171-185, 2010.

[159] S. H. Medina and M. E. H. El-Sayed, "Dendrimers as carriers for delivery of chemotherapeutic agents," Chemical Reviews, vol. 109, no. 7, pp. 3141-3157, 2009.

[160] Q. Xu, C.-H. Wang, and D. W. Pack, "Polymeric carriers for gene delivery: chitosan and poly(amidoamine) dendrimers," Current Pharmaceutical Design, vol. 16, no. 21, pp. 2350-2368, 2010.

[161] R. Huang, L. Han, J. Li et al., "Chlorotoxin-modified macromolecular contrast agent for MRI tumor diagnosis," Biomaterials, vol. 32, no. 22, pp. 5177-5186, 2011.

[162] M. T. Morgan, Y. Nakanishi, D. J. Kroll et al., "Dendrimerencapsulated camptothecins: increased solubility, cellular uptake, and cellular retention affords enhanced anticancer activity in vitro," Cancer Research, vol. 66, no. 24, pp. 1191311921, 2006.

[163] F. Wang, X. Cai, Y. Su et al., "Reducing cytotoxicity while improving anti-cancer drug loading capacity of polypropylenimine dendrimers by surface acetylation," Acta Biomaterialia, vol. 8, no. 12 , pp. 4304-4313.

[164] A. Carlmark, E. Malmstrom, and M. Malkoch, "Dendritic architectures based on bis-MPA: functional polymeric scaffolds for application-driven research," Chemical Society Reviews, vol. 42, no. 13, pp. 5858-5879, 2013.

[165] S. Svenson and A. S. Chauhan, "Dendrimers for enhanced drug solubilization," Nanomedicine, vol. 3, no. 5, pp. 679-702, 2008.

[166] S. Svenson, "Dendrimers as versatile platform in drug delivery applications," European Journal of Pharmaceutics and Biopharmaceutics, vol. 71, no. 3, pp. 445-462, 2009. 
[167] B. Yu, H. C. Tai, W. Xue, L. J. Lee, and R. J. Lee, "Receptortargeted nanocarriers for therapeutic delivery to cancer," Molecular Membrane Biology, vol. 27, no. 7, pp. 286-298, 2010.

[168] P. Kesharwani, V. Gajbhiye, and N. K. Jain, "A review of nanocarriers for the delivery of small interfering RNA," Biomaterials, vol. 33, no. 29, pp. 7138-7150, 2012.

[169] M. Sarikaya, C. Tamerler, A. K.-Y. Jen, K. Schulten, and F. Baneyx, "Molecular biomimetics: nanotechnology through biology," Nature Materials, vol. 2, no. 9, pp. 577-585, 2003.

[170] R. Farr, D. S. Choi, and S. W. Lee, "Phage-based nanomaterials for biomedical applications," Acta Biomaterialia, 2013.

[171] N. F. Steinmetz, "Viral nanoparticles as platforms for nextgeneration therapeutics and imaging devices," Nanomedicine, vol. 6, no. 5, pp. 634-641, 2010.

[172] E. M. Plummer and M. Manchester, "Viral nanoparticles and virus-like particles: platforms for contemporary vaccine design," Wiley Interdisciplinary Reviews, vol. 3, no. 2, pp. 174196, 2011.

[173] M.-A. Poul and J. D. Marks, "Targeted gene delivery to mammalian cells by filamentous bacteriophage," Journal of Molecular Biology, vol. 288, no. 2, pp. 203-211, 1999.

[174] D. Frenkel and B. Solomon, "Filamentous phage as vectormediated antibody delivery to the brain," Proceedings of the National Academy of Sciences of the United States of America, vol. 99, no. 8, pp. 5675-5679, 2002.

[175] T. Nishimoto, K. Yoshida, Y. Miura et al., "Oncolytic virus therapy for pancreatic cancer using the adenovirus library displaying random peptides on the fiber knob," Gene Therapy, vol. 16, no. 5, pp. 669-680, 2009.

[176] S. E. Luria, "Bacteriophage: an essay on virus reproduction," Science, vol. 111, no. 2889, pp. 507-511, 1950.

[177] S. K. Straus, W. R. P. Scott, M. F. Symmons, and D. A. Marvin, "On the structures of filamentous bacteriophage Ff (fd, f1, M13)," European Biophysics Journal, vol. 37, no. 4, pp. 521-527, 2008.

[178] K. Valegard, L. Liljas, K. Fridborg, and T. Unge, "The threedimensional structure of the bacterial virus MS2," Nature, vol. 345, no. 6270, pp. 36-41, 1990.

[179] A. Merzlyak, S. Indrakanti, and S.-W. Lee, "Genetically engineered nanofiber-like viruses for tissue regenerating materials," Nano Letters, vol. 9, no. 2, pp. 846-852, 2009.

[180] S. F. Parmley and G. P. Smith, "Antibody-selectable filamentous fd phage vectors: affinity purification of target genes," Gene, vol. 73, no. 2, pp. 305-318, 1988.

[181] J. K. Scott and G. P. Smith, "Searching for peptide ligands with an epitope library," Science, vol. 249, no. 4967, pp. 386-390, 1990.

[182] G. P. Smith and V. A. Petrenko, "Phage display," Chemical Reviews, vol. 97, no. 2, pp. 391-410, 1997.

[183] Z. M. Carrico, D. W. Romanini, R. A. Mehl, and M. B. Francis, "Oxidative coupling of peptides to a virus capsid containing unnatural amino acids," Chemical Communications, no. 10, pp. 1205-1207, 2008.

[184] R. A. Miller, A. D. Presley, and M. B. Francis, "Self-assembling light-harvesting systems from synthetically modified tobacco mosaic virus coat proteins," Journal of the American Chemical Society, vol. 129, no. 11, pp. 3104-3109, 2007.

[185] N. Stephanopoulos, Z. M. Carrico, and M. B. Francis, "Nanoscale integration of sensitizing chromophores and porphyrins with bacteriophage MS2," Angewandte Chemie, vol. 48, no. 50, pp. 9498-9502, 2009.

[186] T. L. Schlick, Z. Ding, E. W. Kovacs, and M. B. Francis, "Dualsurface modification of the tobacco mosaic virus," Journal of the American Chemical Society, vol. 127, no. 11, pp. 3718-3723, 2005.
[187] G. P. Smith, "Filamentous fusion phage: novel expression vectors that display cloned antigens on the virion surface," Science, vol. 228, no. 4705, pp. 1315-1317, 1985.

[188] R. Pasqualini and E. Ruoslahti, "Organ targeting in vivo using phage display peptide libraries," Nature, vol. 380, no. 6572, pp. 364-366, 1996.

[189] W. Arap, R. Pasqualini, and E. Ruoslahti, "Cancer treatment by targeted drug delivery to tumor vasculature in a mouse model," Science, vol. 279, no. 5349, pp. 377-380, 1998.

[190] D. N. Krag, G. S. Shukla, G.-P. Shen et al., "Selection of tumorbinding ligands in cancer patients with phage display libraries," Cancer Research, vol. 66, no. 15, pp. 7724-7733, 2006.

[191] T. J. Dickerson and K. D. Janda, "Recent advances for the treatment of cocaine abuse: central nervous system immunopharmacotherapy," AAPS Journal, vol. 7, no. 3, pp. E579-E586, 2005.

[192] I. Yacoby, H. Bar, and I. Benhar, "Targeted drug-carrying bacteriophages as antibacterial nanomedicines," Antimicrobial Agents and Chemotherapy, vol. 51, no. 6, pp. 2156-2163, 2007.

[193] D. Frenkel and B. Solomon, "Filamentous phage as vectormediated antibody delivery to the brain," Proceedings of the National Academy of Sciences of the United States of America, vol. 99, no. 8, pp. 5675-5679, 2002.

[194] A. Hajitou, M. Trepel, C. E. Lilley et al., "A hybrid vector for ligand-directed tumor targeting and molecular imaging," Cell, vol. 125, no. 2, pp. 385-398, 2006.

[195] M. E. Martin and K. G. Rice, "Peptide-guided gene delivery," AAPS Journal, vol. 9, no. 1, pp. E18-E29, 2007.

[196] D. Larocca, A. Witte, W. Johnson, G. F. Pierce, and A. Baird, "Targeting bacteriophage to mammalian cell surface receptors for gene delivery," Human Gene Therapy, vol. 9, no. 16, pp. 23932399, 1998.

[197] J. D. Mount, T. I. Samoylova, N. E. Morrison, N. R. Cox, H. J. Baker, and V. A. Petrenko, "Cell targeted phagemid rescued by preselected landscape phage," Gene, vol. 341, no. 1-2, pp. 59-65, 2004.

[198] S. Piersanti, G. Cherubini, Y. Martina et al., "Mammalian cell transduction and internalization properties of $\lambda$ phages displaying the full-length adenoviral penton base or its central domain," Journal of Molecular Medicine, vol. 82, no. 7, pp. 467476, 2004.

[199] S. L. Hart, A. M. Knight, R. P. Harbottle et al., "Cell binding and internalization by filamentous phage displaying a cyclic ArgGly-Asp-containing peptide," Journal of Biological Chemistry, vol. 269, no. 17, pp. 12468-12474, 1994.

[200] M. A. Burg, K. Jensen-Pergakes, A. M. Gonzalez, P. Ravey, A. Baird, and D. Larocca, "Enhanced phagemid particle gene transfer in camptothecin-treated carcinoma cells," Cancer Research, vol. 62, no. 4, pp. 977-981, 2002.

[201] Y. Seow and M. J. Wood, "Biological gene delivery vehicles: beyond viral vectors," Molecular Therapy, vol. 17, no. 5, pp. 767777, 2009.

[202] D. Ghosh, Y. Lee, S. Thomas et al., "M13-templated magnetic nanoparticles for targeted in vivo imaging of prostate cancer," Nature Nanotechnology, vol. 7, no. 10, pp. 677-682, 2012.

[203] M. S. Muthu and B. Wilson, "Multifunctional radionanomedicine: a novel nanoplatform for cancer imaging and therapy," Nanomedicine, vol. 5, no. 2, pp. 169-171, 2010.

[204] J. W. Bulte and M. Modo, Eds., Nanoparticles in Biomedical Imaging: Emerging Technologies and Applications, Springer, New York, NY, USA, 2007. 
[205] D. Janczewski, Y. Zhang, G. K. Das et al., "Bimodal magneticfluorescent probes for bioimaging," Microscopy Research and Technique, vol. 74, no. 7, pp. 563-576, 2011.

[206] P. A. Jarzyna, A. Gianella, T. Skajaa et al., "Multifunctional imaging nanoprobes," Wiley Interdisciplinary Reviews, vol. 2, no. 2, pp. 138-150, 2010.

[207] "Nanoparticles in biomedical imaging, emerging technologies and applications," in Fundamental Biomedical Technologies, M. M. J. M. Jeff and W. M. Bulte, Eds., vol. 102, Springer, New York, NY, USA, 2008.

[208] M. T. McMahon, A. A. Gilad, M. A. DeLiso, S. M. Cromer Berman, J. W. M. Bulte, and P. C. M. Van Zijl, "New "multicolor" polypeptide diamagnetic chemical exchange saturation transfer (DIACEST) contrast agents for MRI," Magnetic Resonance in Medicine, vol. 60, no. 4, pp. 803-812, 2008.

[209] H. Cabral, Y. Matsumoto, K. Mizuno et al., "Accumulation of sub-100 nm polymeric micelles in poorly permeable tumours depends on size," Nature Nanotechnology, vol. 6, no. 12, pp. 815823, 2011. 

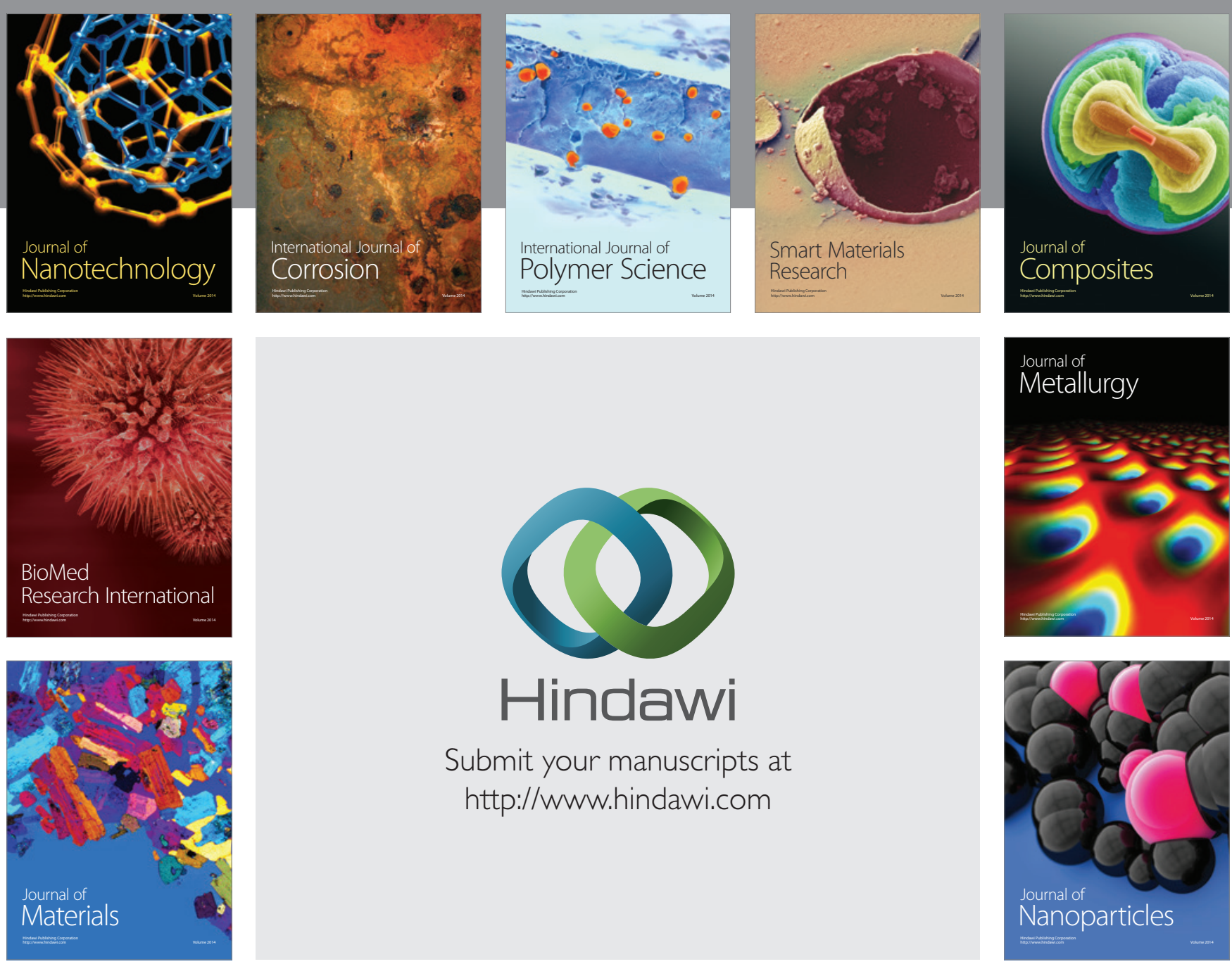

Submit your manuscripts at http://www.hindawi.com
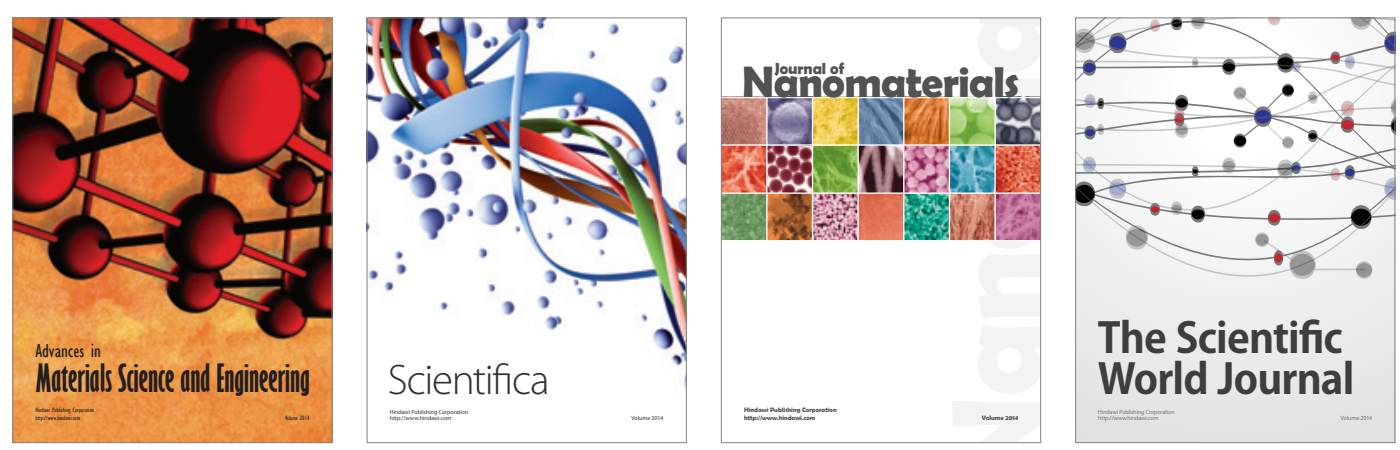

\section{The Scientific World Journal}
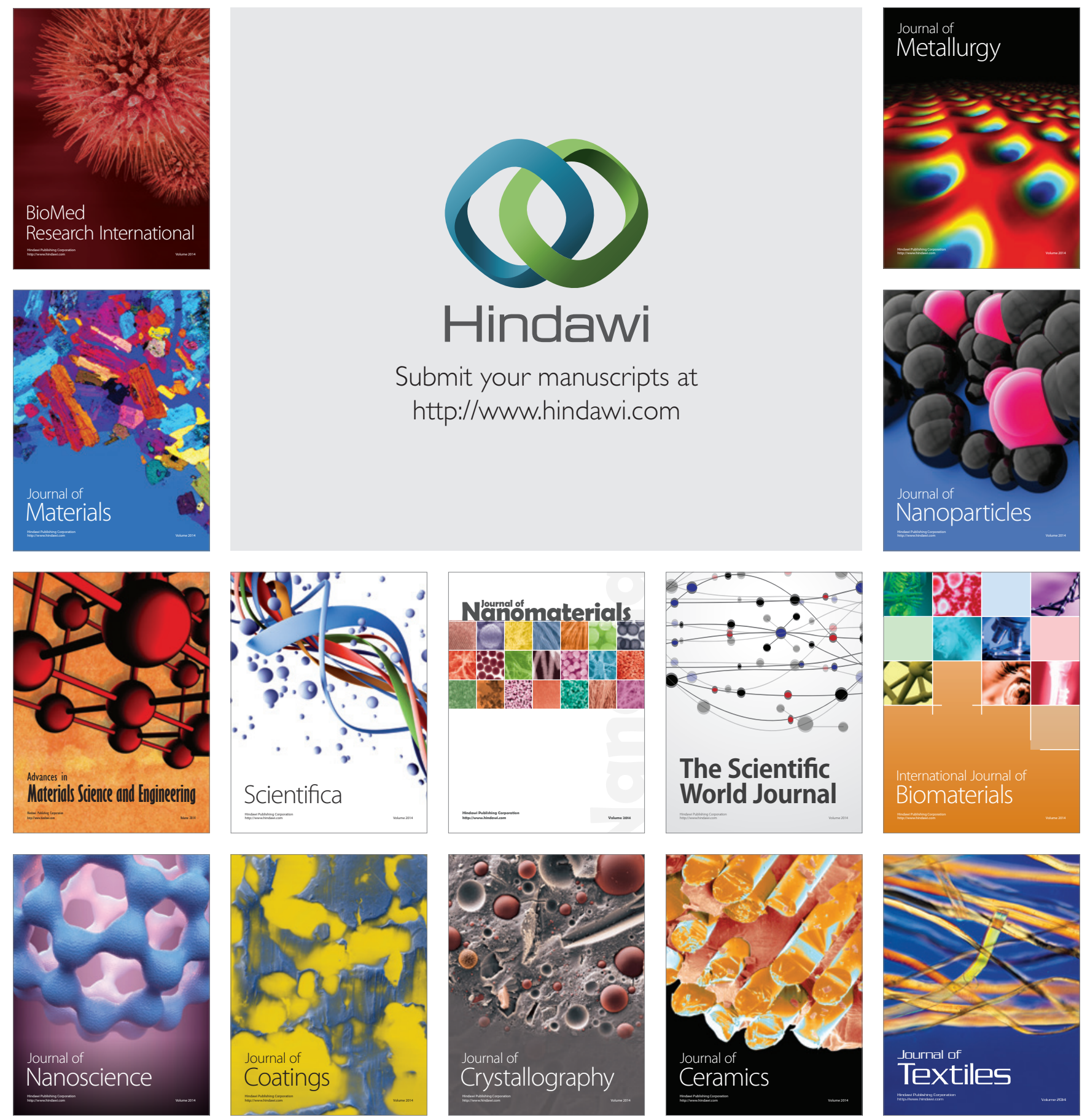